\title{
Variability of the structure and directions in the development of heaths and psammophilous grasslands within the artillery range near the city of Torun
}

\author{
*Joanna Chojnacka, *Wiesław Cyzman, **Andrzej Nienartowicz, **Milosz Deptula \\ *Department of Plant Ecology and Nature Protection, \\ **Laboratory of Ecological Processes Modelling, \\ Institute of Ecology and Protection of Environment, Faculty of Biology and Earth Sciences, \\ Nicolaus Copernicus University, Gagarina 9, 87-100 Toruń, \\ e-mail: anienart@biol.uni.torun.pl
}

\begin{abstract}
Phytosociological analysis of non-forest communities was carried out on one of the oldest and the biggest military training grounds in Europe, located near the city of Torun, in central Poland. Heaths and psammophilous grasslands developed here as a result of deforestation of inland dunes in the valley of the Vistula River, as well as a result of destruction of soils and vegetation brought on by manoeuvres and artillery firings. They form a landscape mosaic with young self-seeding pines, mature pine forests and birch forests. Using the Braun-Blanquet method, 84 relevés were made in the areas dominated by dwarf shrubs and 32 relevés within psammophilous grasslands. Applying the classic phytosociological method, the following plant associations were distinguished in the first set of relevés: Pohlio-Callunetum and Arctostaphylo-Callunetum, as well as the community with Cytisus scoparius. Within the psammophilous grasslands, two associations were distinguished: Spergulo vernalis-Corynephoretum and Calamagrostietum epigeji. In this paper, the heterogeneity within associations and communities was presented together with descriptions of individual syntaxa, as well as dynamic and developmental relationships between the aforementioned were identified.
\end{abstract}

Key words: disturbances, heathlands, inland sand dunes, landscape mosaic, military training area, phytosociology, succession, syntaxonomy, Toruń Valley, Bydgoszcz Forest.

\section{Introduction}

Heathlands, similarly like frequently accompanying psammophilous grasslands, are anthropogenic communities, strictly dependent on human activity. Cessation of land use, consisting in grazing by farm animals, burning and mowing, trigger off succession processes, which quickly bring transformations of grasslands and heaths into fringe, thicket and forest communities. In the conditions of Poland, heaths generally develop on small areas in the landscape of pine forests, less frequently mixed forests. Those are forest glades, unsuccessful forest cultivations, division lines, roadsides, railway embankments etc. Nowadays, larger areas of the aforementioned occur only on military training grounds .

Effects of operations carried out by soldiers during their training courses on training grounds, such as explosions of shells and missiles, fires of forests, damaging the ground and vegetation by tanks, conveyors and artillery guns, building trenches, observation points and battle stations, as well as trampling down, act as inhibitors of the overgrowing process, suspending the succession processes of phyto- and zoocenoses, and ecosystems.

In the territory of Poland, there are seven large training areas of the total area over 90 thousand hectares. The artillery range located near Torun (directly adjacent to the southern limits of the city), is one of the oldest and the 
biggest objects of this type, both in Poland and in Europe. It was already created at the beginning of the 17th century and during the next centuries it was extended, reaching the area of more than 12 thousand hectares (Wasilewski 2004). Since the beginning of the 1990s, the area of the so-called working range, used for training of artillery armed forces, gradually has decreased. At present, besides the decrease of the training area, also the frequency and the intensity of military trainings decrease. Species of shrubs and trees, previously destroyed by military artillery, have begun to encroach on dunes covered with heaths and psammophilous grasslands. The same areas of dunes, which at the beginning of the 1990s were still covered with non-forest vegetation, nowadays constitute a mosaic of pine and birch forests, where only relatively frequent glades, covered with grassland and heath vegetation, remind about the previous exploitation of the training ground (Wasilewski 2004).

Flora and plant communities of the training ground, and especially heaths and psammophilous grasslands, developing here in the conditions of unique human influence, inspired the interest of botanists and ecologists already a long time ago. Botanical observations were conducted here by, inter alia, Wilkoń-Michalska (1964). Gawłowska (1964) and Werdyn (1964) informed that the training ground and the whole Torun Valley are one of the main regions of abundant occurrence of kinnikinnick bearberry and bearberry heaths in Poland. However, difficult accessibility of the area does not create favourable conditions for nature observations on a larger scale. Nowadays, due to changes in the political situation, less frequently due to exploitation and implementation of the EU Programme Natura 2000 in Poland, the area of the training ground is easier to access by naturalists. This creates favourable conditions for understanding the structure and determining the directions of further transformations of ecological systems occurring here. In 1998-2001, the research on protected and rare plant species, as well as plant communities occurring over the whole area of the artillery range were carried out by W. Gugnacka-Fiedor and E. Adamska. The results of this research were presented in a separate paper included in the 12th Issue of Ecological Questions. The main objective of the present paper was to describe the species composition and the structural variability of heaths from the class Nardo-Callunetea occurring in the western part of the artillery range near Torun. In the studied, so-called working part of the artillery range, there are located objects that constitute targets during the artillery firings.

The additional objectives of our research carried out on a smaller spatial scale were to determine the syntaxonomic affiliation of psammophilous grasslands and forest plant communities, which accompany the dwarf shrub heaths, as well as to determine the syndynamic relationships between all distinguished syntaxonomic units. The analyses were conducted with conviction that the research results will provide further information on chorology, as well as spatial and temporal variability of heaths, both in Poland and in Europe.

\section{The study area}

The artillery range stretches out from the city limits of Torun in the north (Fig. 1) till the surroundings of Aleksandrów Kujawski in the south. From the east, it borders on the railway lines Torun - Aleksandrów Kujawski, and from the west - on the road Torun - Gniewkowo Inowrocław. The southern boundary is constituted by the Tążyna rivulet, which runs at the longitude of the village of Otłoczyn, till the village of Grabie and further till the village of Suchatówka along the road leading to the town of Inowrocław. The training ground is in the shape of a pentagon, covers the area of more than 12 thousand hectares, its total length amounts to ca. $16 \mathrm{~km}$, and the width - ca. $13 \mathrm{~km}$ (Wasilewski 2004). Administratively speaking, it is located within the commune of Wielka Nieszawka. Since the area is included in the Forest Division of Gniewkowo, which is under the management of the Regional Administration of State Forests in Torun, it constitutes the property of the State Treasury. The Centre for Artillery and Weaponry Training in Torun is the user of the training ground.

According to the regional, physical and geographical division of Poland, proposed by Kondracki (2000), the training ground is situated within the Province of the Central-European Lowland, the subprovince of Southern-Baltic Lake Districts, the mesoregion of the Torun Valley (also called the Valley of Torun and Bydgoszcz, which is a part of the macroregion of the Torun-Eberswald ice-marginal valley) and the mesoregion of the Inowrocław Plain (the macroregion of the Wielkopolska Lake District). In accordance with the division of Poland into geobotanical regions done by J.M. Matuszkiewicz (1993), the training ground is located in the territory of the Mazovian-Polesie Divide, the Vistula District of Włocławek and Bydgoszcz and the subdistrict of Bydgoszcz Forest (code E1.6.d).

The 400-year history of the training ground is closely connected with the city of Torun, which used to be under the rule of different countries during different periods. Torun was a fortified town, a stronghold, and first of all, a borderland and commercial town, strategically important in campaigns, battles and operations, and the area to the south of the city, together with a bridge abutment protecting the access to the bridge and the city, was the place of fierce battles. Therefore, the city had to maintain relatively substantial defence forces. It was the place where major military forces concentrated, what imposed their training and preparing for wars, mostly with artillery firing. The area was needed to perform those tasks. Beyond the Vistula River, to the south of the city, the present-day central 


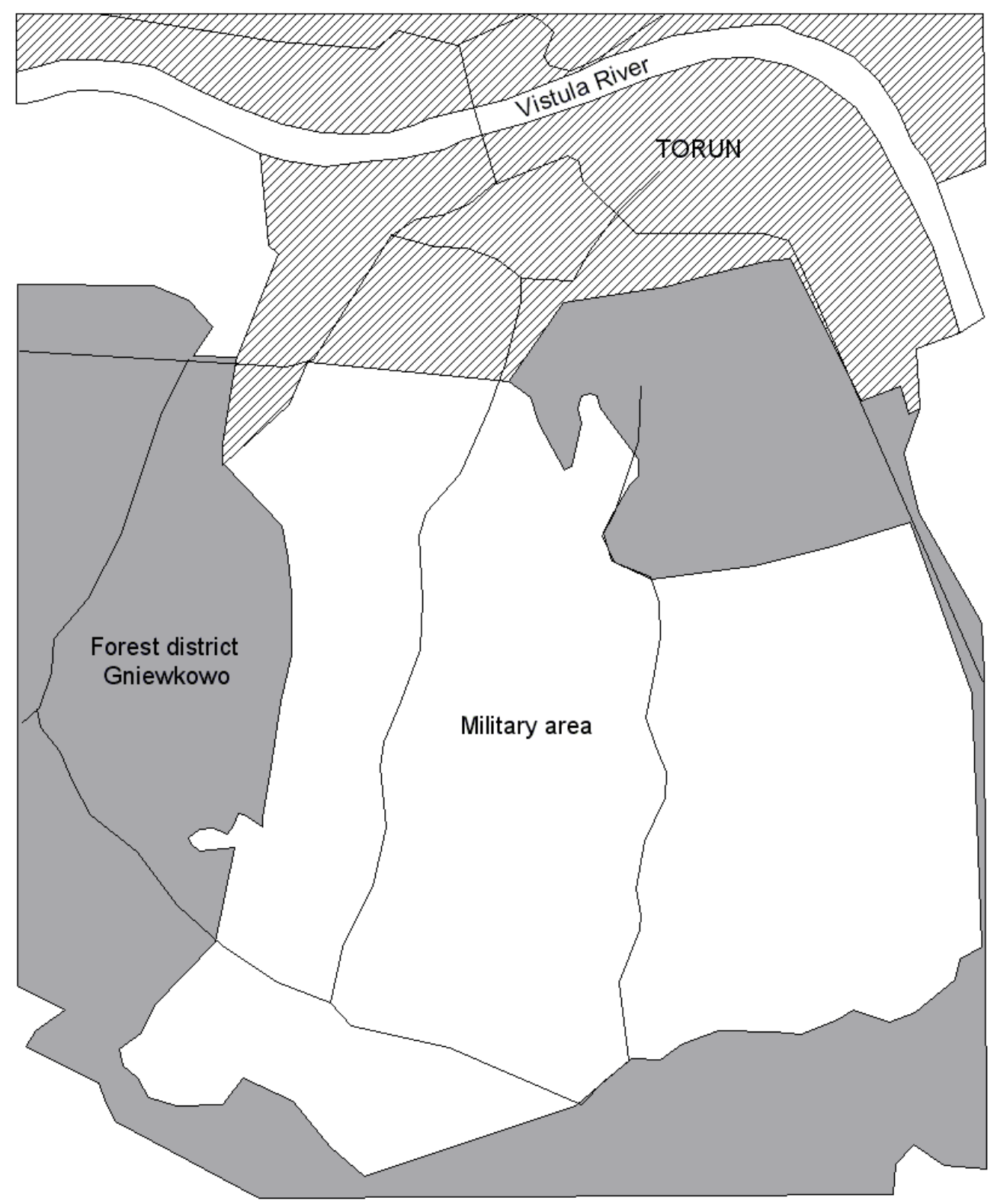

Figure 1. Location of the study area

part of the training ground was not populated due to poor soils. And thus, the area could be used for military trainings (Wasilewski 2004).

The most intensive development of the training ground occurred after 1815, when the area was under the dominion of the Prussian armed forces. After the Prussian-French war in 1871, the infrastructure of the training ground was further extended, using prisoners of war for all the works. At that time, among others, a railway siding was built for ammunition depots, a narrow-gauge railway running along the then boundaries of a working field of the training ground, which was used for distribution of supplies and ammunition. Many hectares of forests growing on those areas were cut down during that time. Until 1884 the area of about 900 ha was developed. During the next years, as a result of the Prussian act on repurchasing the lands, almost 4,900 ha were acquired for military purposes, out of which ca. 2/3 was allotted for the training ground. Already before the 1 st world war, in the training ground located directly at the Prussian-Russian border, there was a training camp for about 20 thousand soldiers. During the 1 st world war, the training ground covered 3,293.0 ha.

In January 1920, Torun together with the adjacent training ground returned under the rule of Poland. In 1921, the 
School of Artillery Firing was opened as a part of the Artillery Training Centre. At that time, the artillery range, taken over by the Polish Army, had the area of 3,890.7 ha.

The latest years before the 2nd World War were characterized by systematic intensification of trainings on the Torun training ground. The encroachment of Wermacht troops in 1939 and the Nazi occupation lasting for the following years, completely changed the nature of this place. At the beginning of the occupation, construction of a training yard for anti-tank artillery commenced. For that reason, hundreds of hectares of forest were cut down exposing bare dunes, which since then were the place of trainings for German soldiers. A number of barracks for German armed forces were built. Also the area was transformed into prisoner-of-war camps and lands of martyrdom for soldiers of many different nationalities. The training ground and the city itself were fortified and incorporated into the zone of strongholds.

Again, after the 2nd World War the area was used as an artillery range for the Polish Armed Forces. During that time, access roads were extended, a working field was protected against fires of grasses and forest, edges of the training ground were marked, the equipment was modernized. The training ground became a base for education and training of artillerists, inter alia, at the Military Academy of Missile and Artillery Forces in Torun. During the 1970s intensive artillery firings proceeded. At that time, several dozen thousand tonnes of ammunition were allocated and stored. During the 1980s and the 1990s the military trainings still took place, but due to financial reasons, they were performed on a smaller area as compared to the previous one (Dzięgielewski 1997).

At present, the training ground makes up 54\% of the Gniewkowo Forest Division, comprising almost entirely its District of Otłoczyn. Forests of the training ground $(5,138.51 \mathrm{ha})$ make up over $34 \%$ of the area of the Gniewkowo Forest Division. Training activity of the armed forces is limited in this area and any exploitation must be preceded by additional arrangements with administration of State Forests. In the land use structure, the largest part of the training ground is constituted by miscellaneous areas $(6,934.26$ ha), on which the Forest Division does not conducts any forest management, and which is earmarked for training sessions, classes and firings by armed forces. This part constitutes a working field of 7,850.90 ha, isolated from the total area of the training ground. In this area, there are training facilities, firing and bombarding zones, fire stations, observation points and technical facilities. After the 2nd World War, the area of the working field within the training ground has slowly decreased. From its northern side, intensive development of the area takes place, especially along the communication routes. During the last few years, at the northern end of the training ground, a clearway was built, which constitutes a southern ring road of Torun.
In the District of Otłoczyn, within the boundaries of which the artillery range is located, two geological and soil formations cover most of the area: eolian formations, dunes and eolian sands, which altogether cover $51.7 \%$ of the total area, as well as fluvial sands of old Pleistocene river terraces covering altogether $36.1 \%$ of the District's area.

Dunes in the Torun-Bydgoszcz Valley form one of the largest inland dune areas in Poland (Galon 1958). According to J. Kobendza and R. Kobendza (1958), the Torun Valley, apart from the ice-marginal valley of the Rivers Warta and Noteć, as well as a junction of the ice-marginal valley of the Rivers Vistula, Bug and Narwia near Warsaw, is one of the most classic dune areas in Europe. Dune areas of the valley are covered by Bydgoszcz Forest. This extensive forest complex is situated within and under the influence of the recommended Bydgoszcz-Torun Metropolitan Area.

More precisely, the area of our research is located within the dune field no. IV (the towns of Torun, Aleksandrów and Gniewkowo), situated on the left shore of the icemarginal valley between the Rivers Zielona and Tążyna. Eolian formations in this part of the Valley occur mainly on the upper, middle (V, VI) and the lower terrace, and in smaller assemblages one can find them also on the alluvial terrace - II (in the village of Nieszawka and the district of Rudak near the city of Toruń).

A characteristic feature of the artillery range near Torun is a poverty of surface waters, which basically do not form any permanent watercourses, reservoirs of stagnant water or wetlands. Water stagnates only for a short period in small depressions between dunes or flows down very quickly infiltrating deep into the soil. However, the supply with rain waters is little. The neighbourhood of Torun is one of the areas with the lowest precipitation in Poland. According to the data from the Base Station in Koniczynka obtained within the framework of Integrated Monitoring of Natural Environment in Poland in 1996-2008, the average annual precipitation was $541.4 \mathrm{~mm}$ and the average annual air temperature was $8.5^{\circ} \mathrm{C}$ (Kejna 2009).

The area of the training ground is characterized by a relatively homogeneous soil cover. Within the training ground, the rusty soil is the dominant soil type covering $83.5 \%$ of the area, and within which the subtype of podzolized soils covers the area of $62.4 \%$. Those are mainly soils developed in eolian sands and in sands of old river terraces. They cover the largest area of the remaining complexes of the Otłoczyn district. In the northern and the central part of the complex, where the research was also carried out, on the tops of dunes or flat surface areas of eolian sands, which are subject to secondary eolian processes, poorly developed soils from loose rocks occur. They cover $4.5 \%$ of the District's area.

The research on the structure, variability and dynamics of heaths and arenaceous grasslands was carried out in 19 


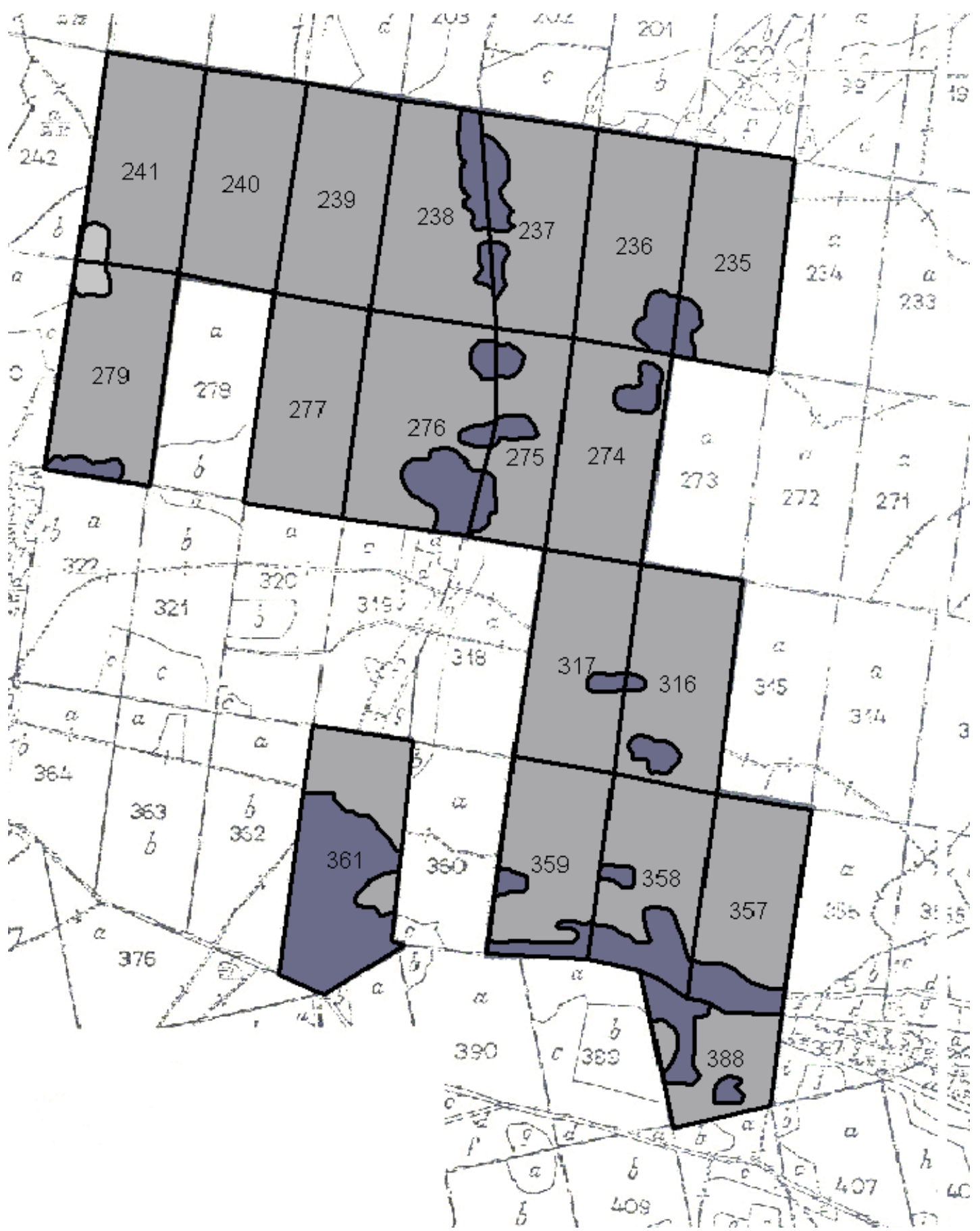

Figure 2. The map of forest sections, in which the research was carried out with the marked soil types (from the soil and habitat survey of the Forest Division of Gniewkowo, 2000)

unforested sections of the Otłoczyn District within the Forest Division of Gniewkowo. Those sections are included in the working training ground. The map of their distribution with the marked soil types according to the soil and habitat Survey of soils and habitats, the Forest Division of Gniewkowo (2000) is presented in Figure 2.

\section{Research methods}

Phytosociological and floristic studies on heathlands of the Torun training ground were performed in 2008, from May till October. The analysis was focused on heath patches selected in the Natura 2000 Programme as a habitat 4030 
(Kujawa-Pawlaczyk 2004), i.e. dry heaths comprising syntaxa Calluno-Genistion, Pohlio-Callunion, Calluno-Arctostaphyllon. Recommendation of natural habitats and objects, as well as field studies were carried out in 2006 and 2007 during a general inventorying of habitats in State Forests of the Gniewkowo Forest Division. During the same period, scientific studies were being conducted also in other nonforest communities of the training ground. The structure of phytocoenoses was described by the generally accepted method of Braun- Blanquet (1951). Altogether, 116 relevés were made, including 84 relevés on heaths and 32 relevés on psammophilous grasslands. The relevés were used for the initial identification of plant communities dominating in the artillery range, and then for their final classification into relevant syntaxonomic units.

Classification of relevés was based on the hierarchical system of plant communities of Poland developed by Matuszkiewicz (2007). Based on the species composition, contribution of differential species and characteristic combination of species, groups of objects were distinguished in the set of relevés, which belong to particular associations and plant communities. Subsets of relevés included in main phytosociological units were compiled into separate tables. Relevés in the tables were divided into units of a lower order, i.e. subassociations and variants. The information included in the tables and in their particular parts were used for further detailed description of all distinguished types of non-forest communities of the study area. A location of each relevé in the field, together with its number in the heading of a relevant table and its affiliation with a specific syntaxon, were presented on the map of the studied fragment of the training ground (Fig. 3).

Apart from the description of heaths and psammophilous grasslands, also a short general description of forest plant communities from the artillery range near Torun was included in this paper. The detailed results of the phytosociological analysis, together with the phytosociological tables, will be presented in a separate report.

\section{The research results}

\subsection{Types of plant communities and their affiliation with higher syntaxonomic units}

The occurrence of two heath associations from the class Nardo-Callunetea was recorded in the studied area. This class also includes the community with Cytisus scoparius of not entirely determined systematic position. Psammophilous grasslands, occurring in the vicinity of heaths, were classified into the association of Spergulo vernalisCorynephoretum from the class Koelerio glaucae-Corynephoretea canescentis and into the association of $\mathrm{Ca}$ - lamagrostietum epigeji from the class Epilobietea angustifolii.

Affiliation of the distinguished communities and associations to higher syntaxonomic units is as follows:

Class: Nardo-Callunetea Prsg 1949

Order: Calluno-Ulicetalia (Quant. 1935) R.Tx. 1937

1. The community with Cytisus scoparius

Alliance: Pohlio-Callunion Shimwell 1973 em. Brzeg 1981

2. Pohlio-Callunetum Shimwell 1973 em. Brzeg 1981 Alliance: Calluno-Arctostaphylion R.Tx. et Prsg 1949

3. Arctostaphylo-Callunetum R.Tx. et Prsg 1940

Class: Koelerio glaucae-Corynephoretea canecentis Klika in Klika et Novak 1941

Order: Corynephoretalia canescentis R.Tx. 1937

Alliance: Corynephorion canescentis Klika 1934

4. Spergulo vernalis-Corynephoretum (R. Tx. 1928) Libb. 1933

Class: Epilobietea angustifolii R.Tx. et Prsg 1950

Order: Atropetalia Vlieg. 1937

Alliance: Epilobion angustifolii (Rubel 1933) Soo 1933

5. Calamagrostietum epigeji Juraszek 1928

\subsection{Characteristics and diversity of heaths}

Heath communities from the class Nardo-Callunetea occur in the whole studied area, in many places as a mosaic with psammophilous grasslands or pine forests and „birch groves”. Most of the phytocoenoses dominated by Calluna vulgaris were classified as heaths with nodding thread-moss from the association Pohlio-Callunetum. Few and small clusters of bearberry heaths concentrate in the north-western part of the studied area, mainly in the section 279 of the Otłoczyn forest district. Whereas the community with Cytisus scoparius occurs most frequently in the southern part of the studied area.

The structure of phytocoenoses classified into particular syntaxa is presented in relevés included in Tables 1-3. The comparison of relative cover of species from different syngenetic groups of five distinguished syntaxa is presented in Figure 4.

\subsubsection{Association Pohlio-Callunetum}

Patches of heaths with nodding thread-moss Pohlio-Callunetum of different size are scattered all over the studied fragment of the artillery range near Torun. Its patches occur both on slopes of dunes with different inclinations and in flat areas and in depressions. They neighbour on fragments of fresh coniferous forest Peucedano-Pinetum, grasslands with gray clubawn grass Spergulo vernalisCorynephoretum, the community with chee reedgrass and common groom. 


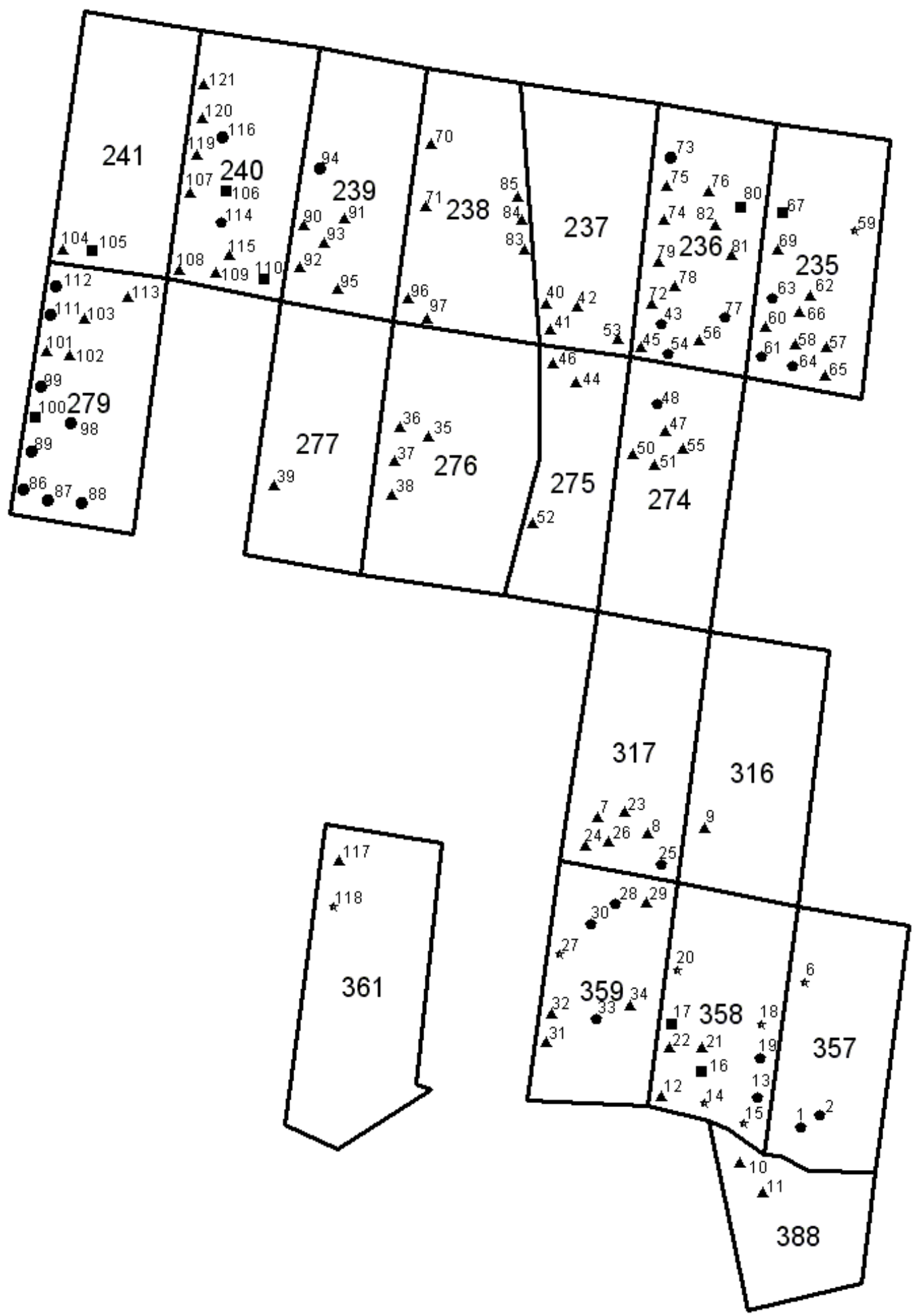

Figure 3. The map of forest sections with the marked sites of relevés 


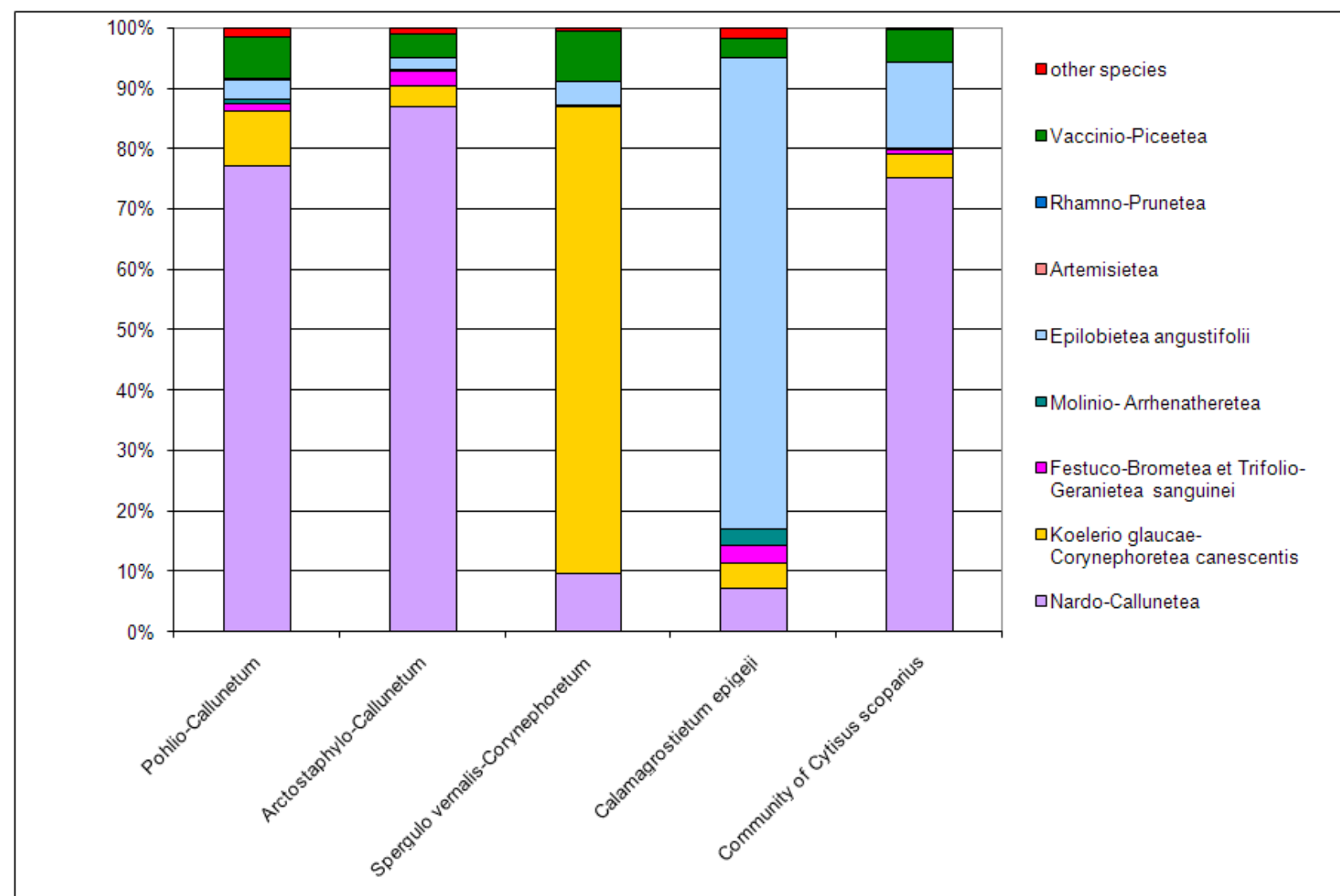

Figure 4. A relative cover degree of particular vegetation classes (syngenetic groups of species) in non-forest communities of the artillery range near the city of Torun

Patches of Pohlio-Callunetum occur in the form of a community of dwarf shrubs, distinguished by the domination of Scots heather Calluna vulgaris, assuming most frequently a facial form. The structure of the association is two-layered. The higher layer is composed almost exclusively of Calluna vulgaris, whereas the lower one is dominated by much shorter plants, such as e.g.: yellow hawkweed Hieracium pilosella, wild thyme Thymus serpyllum or heath sedge Carex ericetorum. In some patches, forest succession proceeds, and this is expressed by the presence of seedlings and undergrowth trees, especially of Scots pine Pinus sylvestris and European white birch Betula pendula.

73 relevés were classified into the association PohlioCallunetum (Tab. 1). They are diversified into two variants: typical and with clusterhead pink Dianthus carthusianorum.

The typical variant is more species poor. It is mainly characterized by strong domination of Calluna vulgaris. Apart from the aforementioned species, the herb layer is mostly composed of: Carex ericetorum, Hieracium pilosella, H. umbellatum from the order Calluno-Ulicetalia and from the class Nardo-Callunetea. Among the species characteristic and differential of the association Pohlio-Callunetum and the alliance Pohlio-Callunion, certain species of mosses and lichens occur with small quantities: Pohlia nutans, Dicranum scoparium, Cladonia chlorophaea s. 1., C. coniocraea and $C$. deformis. Also accompanying species from the class Koelerio glaucae-Corynephoretea canescentis are very significant in the typical variant, e.g. Corynephorus canescens, Thymus serpyllum, and from the class Vaccinio-Piceetea, mainly Deschampsia flexuosa.

In the patches classified into the typical variant, the form with Pleurozium schreberi was distinguished (relevés 67-73, Tab. 1). Its phytocoenoses cover shaded places of northern or north-eastern exposure, usually in the vicinity of coniferous forests. They are characterized by relatively high contribution of the aforementioned moss species and other species of vascular plants, mosses and lichens from the class Vaccinio-Piceetea, such as: Deschampsia flexuosa, Dicranum undulatum, Cladonia squamosa.

The variant with Dianthus carthusianorum is significantly richer in species than the typical variant. It is dis- 
Table 1 (the first part). The association Pohlio-Callunetum (relevés 1-10, the variant with Dianthus carthusianorum, the subvariant with Molinia caerulea)

\begin{tabular}{|l|c|c|c|c|c|c|c|c|c|c|c|}
\hline \multicolumn{1}{|c|}{ Relevé number in table } & 1 & 2 & 3 & 4 & 5 & 6 & 7 & 8 & 9 & 10 & Constancy \\
\hline Relevé number & 60 & 66 & 82 & 56 & 93 & 35 & 81 & 103 & 58 & 65 & \\
\hline Date & $\begin{array}{r}16.07 . \\
2008\end{array}$ & $\begin{array}{c}16.07 . \\
2008\end{array}$ & $\begin{array}{c}17.07 . \\
2008\end{array}$ & $\begin{array}{c}16.07 . \\
2008\end{array}$ & $\begin{array}{c}17.07 . \\
2008\end{array}$ & $\begin{array}{c}25.06 . \\
2008\end{array}$ & $\begin{array}{c}17.07 . \\
2008\end{array}$ & $\begin{array}{c}18.08 . \\
2008\end{array}$ & $\begin{array}{c}16.07 . \\
2008\end{array}$ & $\begin{array}{c}16.07 . \\
2008\end{array}$ \\
\hline Forest section & 235 & 235 & 236 & 236 & 239 & 276 & 236 & 279 & 235 & 235 \\
\hline Relevé area (m) & $20 \times 20$ & $10 \times 10$ & $20 \times 20$ & $20 \times 20$ & $20 \times 20$ & $20 \times 20$ & $10 \times 10$ & $20 \times 20$ & $20 \times 20$ & $10 \times 10$ \\
\hline Slope (\%) & & & & & & & 10 & 20 & & 10 \\
\hline Exposure & & & & & & & $\mathrm{E}$ & $\mathrm{N}$ & & E \\
\hline Density of layer a (\%) & 5 & & 5 & 5 & 5 & & & & 5 & 5 \\
\hline Density of layer b (\%) & 5 & & 5 & 5 & 5 & & & & 5 & \\
\hline Cover of layer c (\%) & 90 & 90 & 90 & 90 & 90 & 90 & 90 & 90 & 90 & 90 \\
\hline Cover of layer d (\%) & 20 & 10 & 10 & 10 & 10 & 10 & 20 & 20 & 10 & 10 \\
\hline Number of species in relevé & 25 & 24 & 22 & 23 & 23 & 20 & 20 & 25 & 21 & 24 \\
\hline
\end{tabular}

Ch. D. Pohlio-Callunetum et Pohlio-Callunion

\begin{tabular}{|l|c|c|c|c|c|c|c|c|c|c|c|}
\hline Pohlia nutans & + & & + & & 1 & & & & + & 1 & III \\
\hline Dicranum scoparium & + & & & & + & + & & + & + & + & II \\
\hline Cladonia chlorophaea s. 1 & & + & + & & 1 & & + & + & & + & IV \\
\hline Cladonia coniocraea & & & & & & & 1 & & & & II \\
\hline Cladonia deformis & + & + & & + & & & & & + & + & II \\
\hline
\end{tabular}

Ch. D. Arctostaphylo-Callunetum et Calluno-Arctostaphylion:

\begin{tabular}{|l|c|c|c|c|c|c|c|c|c|c|c|}
\hline Carex ericetorum & 1 & 1 & + & + & 1 & & & + & 2 & + & $\mathrm{V}$ \\
\hline Peucedanum oreoselinum & 1 & + & + & + & + & + & + & 1 & 1 & + & $\mathbf{V}$ \\
\hline Solidago virgaurea & + & + & + & + & & + & + & 1 & + & + & $\mathbf{V}$ \\
\hline Polygonatum odoratum & & + & & & + & & & & + & & $\mathbf{I}$ \\
\hline
\end{tabular}

Ch. D. Calluno-Ulicetalia et Nardo-Callunetea:

\begin{tabular}{|l|c|c|c|c|c|c|c|c|c|c|c|}
\hline Calluna vulgaris & 4 & 2 & 3 & 5 & 3 & 4 & 4 & 4 & 4 & 3 & V \\
\hline Hieracium pilosella & & 1 & + & + & 1 & 1 & 1 & 3 & 1 & + & V \\
\hline Hieracium umbellatum & & & + & + & + & & + & & + & II \\
\hline Viola canina & & + & & + & & & & & & & I \\
\hline Veronica officinalis & & & & 1 & & & & & & + & I \\
\hline
\end{tabular}

Accompanying species:

a) $\mathrm{Cl}$. Koelerio glaucae-Corynephoretea canescentis

\begin{tabular}{|l|c|c|c|c|c|c|c|c|c|c|c|}
\hline Corynephorus canescens & + & 1 & 1 & 1 & 1 & + & 2 & + & 1 & 1 & V \\
\hline Thymus serpyllum & 1 & & + & 1 & & & 1 & 3 & 1 & + & III \\
\hline Jasione montana & + & + & & & + & & + & & + & + & II \\
\hline Scleranthus perennis & & + & & & & + & & & & & I \\
\hline
\end{tabular}




\begin{tabular}{|l|c|c|c|c|c|c|c|c|c|c|c|}
\hline \multicolumn{1}{|c|}{ Relevé number in table } & 1 & 2 & 3 & 4 & 5 & 6 & 7 & 8 & 9 & 10 & Constancy \\
\hline Festuca ovina & & & & & 1 & 1 & & & & II \\
\hline Helichrysum arenarium & & + & & & & & & & & I \\
\hline Potentilla argentea & & 1 & & & & & & & & I \\
\hline Rumex acetosella & + & & & & & & & + & & + & I \\
\hline Brachythecium albicans & & & & + & & & & + & & & I \\
\hline Ceratodon purpureus & + & + & & + & & & + & + & & & III \\
\hline Polytrichum piliferum & + & + & & + & & & + & + & + & 1 & IV \\
\hline Cladonia macilenta & + & & + & + & & + & + & & & & III \\
\hline Cladonia cervicornis & & & & + & & & & & & & I \\
\hline $\begin{array}{l}\text { Cladonia arbuscula subsp. } \\
\text { mitis }\end{array}$ & + & + & + & & & + & + & & & IV \\
\hline Cladonia uncialis & & & + & & + & & + & & & & I \\
\hline
\end{tabular}

b) Cl. Festuco-Brometea et Trifolio-Geranietea:

\begin{tabular}{|l|c|c|c|c|c|c|c|c|c|c|c|}
\hline Galium verum & & & & & & 1 & + & & & I \\
\hline Dianthus carthusianorum & + & + & + & & + & & + & 1 & & & II \\
\hline Euphorbia cyparissias & & & & & & 1 & & 1 & & & II \\
\hline Potentilla arenaria & & & & & & & & 2 & & & I \\
\hline Geranium sanguineum & & & & + & & & & & & II \\
\hline
\end{tabular}

c) Cl. Molinio-Arrhenatheretea:

\begin{tabular}{|l|c|c|c|c|c|c|c|c|c|c|c|}
\hline Rumex acetosa & & & & & & & & + & & & I \\
\hline Molinia caerulea & 1 & 1 & + & 1 & + & + & + & + & 1 & 1 & I \\
\hline
\end{tabular}

d) Cl. Epilobietea angustifolii:

\begin{tabular}{|l|c|c|c|c|c|c|c|c|c|c|c|}
\hline Calamagrostis epigejos & + & 2 & + & + & & + & 1 & 1 & 1 & + & V \\
\hline Rubus idaeus & & & & & & 1 & & & & & I \\
\hline
\end{tabular}

e) Cl. Artemisietea:

\begin{tabular}{|c|c|c|c|c|c|c|c|c|c|c|}
\hline Linaria vulgaris & & + & & & & & & & & I \\
\hline Verbascum densiflorum & & & & & & $\mathrm{r}$ & & & & I \\
\hline \multicolumn{11}{|c|}{ f) Species of Rhamno-Prunetea: } \\
\hline Crataegus monogyna $b$ & & & & & & & $\mathrm{r}$ & & & I \\
\hline \multicolumn{11}{|l|}{ g) Cl. Vaccinio-Piceetea: } \\
\hline Pinus sylvestris a & 1 & & 1 & & 1 & & & & 1 & $\mathbf{I}$ \\
\hline Pinus sylvestris $b$ & & & + & & & & & + & & $\mathbf{I}$ \\
\hline Pinus sylvestris c & & & & & + & & & & & II \\
\hline Deschampsia flexuosa & & & 1 & & 1 & 1 & 1 & + & & III \\
\hline Pleurozium schreberi & & & & + & & & & & & II \\
\hline Dicranum undulatum & + & & & & & & & & + & II \\
\hline
\end{tabular}




\begin{tabular}{|l|c|c|c|c|c|c|c|c|c|c|c|}
\hline \multicolumn{1}{|c|}{ Relevé number in table } & 1 & 2 & 3 & 4 & 5 & 6 & 7 & 8 & 9 & 10 & Constancy \\
\hline Cladonia rangiferina & + & & + & & + & & & & & + & II \\
\hline Cladonia furcata & + & & + & & & & + & + & & II \\
\hline $\begin{array}{l}\text { Cladonia arbuscula subsp. } \\
\text { arbuscula }\end{array}$ & & & & & & & & & & & I \\
\hline Cladonia gracilis & & & & + & + & & & & + & & II \\
\hline Cladonia fimbriata s. 1. & & & & & & & & + & & + & I \\
\hline Cladonia digitata & & + & & & & & & + & & & I \\
\hline
\end{tabular}

h) other species:

\begin{tabular}{|l|c|c|c|c|c|c|c|c|c|c|c|}
\hline Betula pendula a & & & & 1 & & & & & 1 & 1 & II \\
\hline Betula pendula b & $\mathrm{r}$ & & & + & & & & & & & II \\
\hline Betula pendula c & & & & & + & & & & + & + & II \\
\hline Populus tremula c & & & & & & + & & & & & I \\
\hline Pyrus communis c & & & & & $\mathrm{r}$ & + & & & & & I \\
\hline Hypericum perforatum & + & + & & & + & & & + & & & II \\
\hline Sciuro-hypnum starkei & & & + & & & & & & & & I \\
\hline Polytrichum juniperinum & + & + & & & & + & & & & + & III \\
\hline Cetraria ericetorum & & & & & & & & + & & & I \\
\hline
\end{tabular}


Table 1 (the second part). The association Pohlio-Callunetum (relevés 11-22, the variant with Dianthus carthusianorum, the typical subvariant, the form with Pleurozium schreberi)

\begin{tabular}{|l|c|c|c|c|c|c|c|c|c|c|c|c|}
\hline \multicolumn{1}{|c|}{ Relevé number in table } & 11 & 12 & 13 & 14 & 15 & 16 & 17 & 18 & 19 & 20 & 21 & 22 \\
\hline Relevé number & 95 & 96 & 72 & 85 & 47 & 101 & 84 & 120 & 45 & 57 & 102 & 53 \\
\hline Date & $\begin{array}{c}17.07 . \\
2008\end{array}$ & $\begin{array}{c}17.07 . \\
2008\end{array}$ & $\begin{array}{c}17.07 . \\
2008\end{array}$ & $\begin{array}{c}17.07 . \\
2008\end{array}$ & $\begin{array}{c}7.07 . \\
2008\end{array}$ & $\begin{array}{c}18.08 \\
2008\end{array}$ & $\begin{array}{c}17.07 . \\
2008\end{array}$ & $\begin{array}{c}10.10 . \\
2008\end{array}$ & $\begin{array}{c}7.07 . \\
2008\end{array}$ & $\begin{array}{c}16.07 . \\
2008\end{array}$ & $\begin{array}{c}18.08 . \\
2008\end{array}$ & $\begin{array}{c}16.07 . \\
2008\end{array}$ \\
\hline Forest section & 239 & 238 & 236 & 238 & 274 & 279 & 238 & 240 & 236 & 235 & 279 & 237 \\
\hline Relevé area (m) (m) & $10 / 10$ & $10 / 10$ & $10 / 10$ & $20 / 20$ & $20 / 20$ & $20 / 20$ & $20 / 20$ & $20 / 20$ & $10 / 10$ & $20 / 20$ & $20 / 20$ & $20 / 20$ \\
\hline Slope ( ) & & & & 20 & & 40 & & & & & 40 & \\
\hline Exposure & & & & $\mathrm{N}$ & & $\mathrm{S}$ & & & & & $\mathrm{N}$ & \\
\hline Density of layer a (\%) & 5 & & & 10 & 5 & 10 & & 5 & 5 & 5 & 5 & 5 \\
\hline Density of layer b (\%) & 5 & & & & 5 & 5 & & 30 & 5 & 5 & 5 & 5 \\
\hline Cover of layer c (\%) & 90 & 90 & 90 & 90 & 90 & 90 & 90 & 80 & 90 & 90 & 90 & 80 \\
\hline Cover of layer d (\%) & 10 & 20 & 10 & 10 & 10 & 30 & 20 & 10 & 20 & 10 & 10 & 10 \\
\hline Number of species in relevé & 27 & 24 & 29 & 26 & 28 & 35 & 25 & 20 & 28 & 26 & 22 & 25 \\
\hline
\end{tabular}

Ch. D. Pohlio-Callunetum et Pohlio-Callunion

\begin{tabular}{|c|c|c|c|c|c|c|c|c|c|c|c|}
\hline Pohlia nutans & & 1 & + & & & & & & & & \\
\hline Dicranum scoparium & + & + & & 1 & + & + & + & & + & + & + \\
\hline Cladonia chlorophaea s. 1. & & & & & & & 1 & + & + & & \\
\hline Cladonia coniocraea & 1 & & & & & & & 1 & & & 1 \\
\hline Cladonia deformis & & + & & & & & & & + & & + \\
\hline Agrostis capillaris & & & & & + & & & & & & \\
\hline
\end{tabular}

Ch. D. Arctostaphylo-Callunetum et Calluno-Arctostaphylion:

\begin{tabular}{|l|c|c|c|c|c|c|c|c|c|c|c|c|}
\hline Carex ericetorum & 1 & 1 & + & 1 & 1 & + & 1 & & & 1 & + \\
\hline Peucedanum oreoselinum & + & + & + & + & 1 & 2 & + & 1 & 1 & + & 1 & 1 \\
\hline Solidago virgaurea & + & + & + & + & & + & 1 & & 1 & + & + & + \\
\hline Vincetoxicum hirundinaria & 1 & & & & + & 1 & & & & & & \\
\hline Polygonatum odoratum & & & & & & & & & & & + & \\
\hline Ch. D. Calluno-Ulicetalia et Nardo-Callunetea: & 3 & 4 & 3 & 4 & 3 & 2 & 4 & 4 & 2 & 4 & 3 & 4 \\
\hline Calluna vulgaris & 1 & + & + & + & & 2 & + & 1 & & 2 & & + \\
\hline Hieracium pilosella & & + & + & + & & & & & & & & \\
\hline Hieracium umbellatum & + & & & & & + & & + & & & & \\
\hline Viola canina & & & & & & & & & & & & \\
\hline Veronica officinalis & 11 & 12 & 13 & 14 & 15 & 16 & 17 & 18 & 19 & 20 & 21 & 22 \\
\hline Relevé number in table & & & & & & & & & & & & \\
\hline Potentilla erecta & & & & & & & & & \\
\hline Cytisus scoparius & & & & & & & & \\
\hline Accompanying species:
\end{tabular}




\begin{tabular}{|c|c|c|c|c|c|c|c|c|c|c|c|c|}
\hline Relevé number in table & 11 & 12 & 13 & 14 & 15 & 16 & 17 & 18 & 19 & 20 & 21 & 22 \\
\hline \multicolumn{13}{|c|}{ a) Cl. Koelerio glaucae-Corynephoretea canescentis } \\
\hline Corynephorus canescens & & 1 & 1 & + & 1 & 2 & 1 & & + & 2 & + & 1 \\
\hline Thymus serpyllum & 1 & 1 & 1 & + & + & 1 & 1 & + & & & & + \\
\hline Jasione montana & + & & & + & & & & + & + & 1 & & \\
\hline Scleranthus perennis & & & + & & + & & + & & & & & \\
\hline Festuca ovina & + & & + & 1 & & & & & 1 & & & \\
\hline \multicolumn{13}{|l|}{ Spergula vernalis } \\
\hline Helichrysum arenarium & & & & & 1 & & & + & & & & \\
\hline Potentilla argentea & & & + & & & & & + & + & & & \\
\hline Rumex acetosella & & & & & & & & & & + & & \\
\hline \multicolumn{13}{|l|}{ Koeleria glauca } \\
\hline \multicolumn{13}{|l|}{$\begin{array}{l}\text { Lotus corniculatus var. } \\
\text { crassifolius }\end{array}$} \\
\hline Astragalus arenarius & & & & & & & & & & & & + \\
\hline \multicolumn{13}{|l|}{ Agrostis vinealis } \\
\hline \multicolumn{13}{|l|}{ Carex arenaria } \\
\hline Brachythecium albicans & & + & & & & & + & & & + & & \\
\hline Ceratodon purpureus & & + & & & + & + & & + & + & + & & \\
\hline Polytrichum piliferum & & & + & & & + & + & & + & & & + \\
\hline Cladonia macilenta & & + & & & + & + & + & & & & + & \\
\hline Cladonia cervicornis & & & & & & & & + & & & & \\
\hline $\begin{array}{l}\text { Cladonia arbuscula subsp. } \\
\text { mitis }\end{array}$ & & & + & + & + & + & + & & + & & + & + \\
\hline Cladonia uncialis & & + & + & & + & & & & & & & \\
\hline Cladonia phyllophora & & & & & + & & & & & & & \\
\hline \multicolumn{13}{|c|}{ b) Cl. Festuco-Brometea et Trifolio-Geranietea: } \\
\hline Galium verum & & + & 1 & 1 & & & + & & + & & & \\
\hline Dianthus carthusianorum & + & & + & + & + & 1 & + & & & & & \\
\hline Euphorbia cyparissias & + & & 1 & 1 & + & + & & + & & & + & \\
\hline Anthericum ramosum & + & + & + & + & + & & & & & & & + \\
\hline Potentilla arenaria? & + & & & & & 1 & & + & & & & \\
\hline Achillea pannonica & + & & + & & & & & & & & & \\
\hline \multicolumn{13}{|l|}{ Silene nutans } \\
\hline \multicolumn{13}{|l|}{ Lathyrus sylvestris } \\
\hline \multicolumn{13}{|l|}{ Potentilla neumanniana } \\
\hline Geranium sanguineum & + & & & & & & & & & & & \\
\hline
\end{tabular}




\begin{tabular}{|l|l|l|l|l|l|l|l|l|l|l|l|l|}
\hline \multicolumn{1}{|c|}{ Relevé number in table } & 11 & 12 & 13 & 14 & 15 & 16 & 17 & 18 & 19 & 20 & 21 & 22 \\
\hline Carlina vulgaris & & & & & & & + & & & & & \\
\hline
\end{tabular}

c) Cl. Molinio-Arrhenatheretea:

\begin{tabular}{|l|l|l|l|l|l|l|l|l|l|l|l|l|}
\hline Rumex acetosa & & & & & & 2 & & & & & 1 & \\
\hline Achillea millefolium & & & 1 & & & & & & + & & & \\
\hline Molinia caerulea & & & & & & & & & & & & \\
\hline Knautia arvensis & & & & & & + & & & & + & & \\
\hline Sanguisorba officinalis & & & & & & & & & & & & \\
\hline Dianthus superbus & & & & & & & & & & & & \\
\hline
\end{tabular}

d) Cl. Epilobietea angustifolii:

\begin{tabular}{|l|l|l|l|l|l|l|l|l|l|l|l|l|}
\hline Calamagrostis epigejos & 2 & & 1 & + & 1 & 1 & & 1 & & 1 & 1 & \\
\hline Rubus idaeus & & & & & & & & & & & & \\
\hline Epilobium angustifolium & & & & & & & & & & & & \\
\hline
\end{tabular}

e) Cl. Artemisietea:

Artemisia vulgaris

Linaria vulgaris

Verbascum densiflorum

f) $\mathrm{Cl}$. Rhamno-Prunetea:

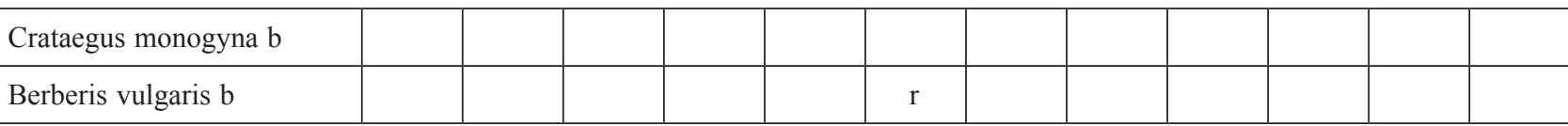

g) Cl. Vaccinio-Piceetea:

\begin{tabular}{|l|l|l|l|l|l|l|l|l|l|l|l|l|}
\hline Pinus sylvestris a & & & & & & 1 & & & 1 & 1 & 1 \\
\hline Pinus sylvestris b & & & & & & + & & & + & & + \\
\hline Pinus sylvestris c & + & & + & & & + & + & & & $\mathrm{r}$ & \\
\hline Convallaria majalis & & & & & & & & & + & & 1 & \\
\hline Deschampsia flexuosa & & 1 & 1 & 1 & 1 & 2 & + & & 2 & 1 & 2 & 1 \\
\hline Vaccinium vitis-idaea & & & & 1 & & & & & 2 & & 1 & \\
\hline Vaccinium myrtillus & & & & + & & & & & & & & \\
\hline Calamagrostis arundinacea & & & & & & & & & & & & \\
\hline Pleurozium schreberi & 1 & 2 & 1 & 2 & 1 & 2 & 1 & 1 & 1 & 1 & 1 & 1 \\
\hline Dicranum undulatum & & & + & + & + & + & 1 & + & + & + & + & + \\
\hline Cladonia rangiferina & & & & & & + & & + & + & & + & + \\
\hline Cladonia furcata & & & & & & & & & & + & + \\
\hline $\begin{array}{l}\text { Cladonia arbuscula subsp. } \\
\text { arbuscula }\end{array}$ & & & + & & & + & & & + & + & + & + \\
\hline Cladonia gracilis & & + & + & & + & & + & + & + & + & + \\
\hline Cladonia fimbriata s. 1. & & & & & & & & & & + \\
\hline
\end{tabular}




\begin{tabular}{|l|c|c|c|c|c|c|c|c|c|c|c|c|}
\hline \multicolumn{1}{|c|}{ Relevé number in table } & 11 & 12 & 13 & 14 & 15 & 16 & 17 & 18 & 19 & 20 & 21 & 22 \\
\hline Cladonia squamosa & + & + & & + & & + & + & & + & & & + \\
\hline Cladonia digitata & & & & & & & & & & & & \\
\hline
\end{tabular}

h) other species:

\begin{tabular}{|c|c|c|c|c|c|c|c|c|c|c|c|c|}
\hline Betula pendula a & 1 & & & & 1 & 1 & & & & & & 1 \\
\hline Betula pendula $\mathrm{b}$ & + & & & & & & & + & & & + & $\mathrm{r}$ \\
\hline Betula pendula c & & + & & & & + & & & & & + & \\
\hline Populus tremula b & & & & & & & & + & & $\mathrm{r}$ & & \\
\hline Populus tremula c & & & & & & & & & & 1 & & \\
\hline \multicolumn{13}{|l|}{ Prunus padus $b$} \\
\hline \multicolumn{13}{|l|}{ Pyrus communis $\mathrm{c}$} \\
\hline Hypericum perforatum & + & & + & & + & & & & & + & & + \\
\hline \multicolumn{13}{|l|}{ Hypochoeris radicata } \\
\hline \multicolumn{13}{|l|}{ Trifolium medium } \\
\hline \multicolumn{13}{|l|}{ Senecio vulgaris } \\
\hline Sorbus aucuparia c & & & & & & & & & & & & $\mathrm{r}$ \\
\hline Carlina acaulis & & & & & & $\mathrm{r}$ & & & & & & \\
\hline Polypodium vulgare & & & & & & & & & & & + & \\
\hline Trifolium lupinaster & & & & & & + & & & & & & \\
\hline Quercus petreae c & & & & & & & & & + & & & $\mathrm{r}$ \\
\hline Sciuro-hypnum starkei & & & & + & & & & & + & & & \\
\hline Sciuro-hypnum oedipodium & + & & & + & & + & + & & + & & & \\
\hline Oxyrrhynchium hians & & & & + & & & & + & & & & \\
\hline Polytrichum juniperinum & + & & & & & & & & & & & \\
\hline Peltigera rufescens & & + & & & & & + & & & & & \\
\hline Cetraria ericetorum & & + & & & + & & & & & & & \\
\hline
\end{tabular}




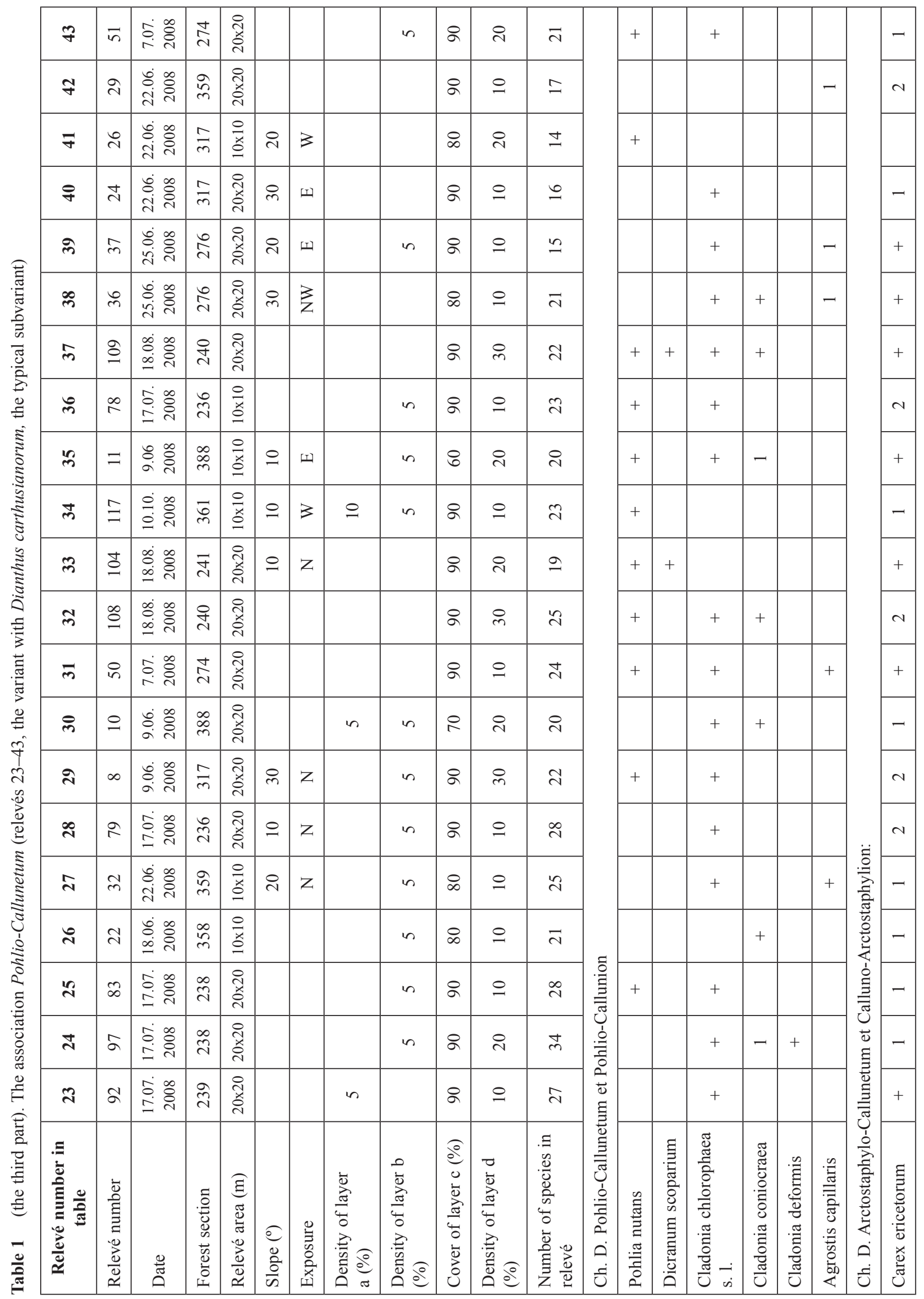




\begin{tabular}{|c|c|c|c|c|c|c|c|c|c|c|c|c|c|c|c|c|c|c|c|c|c|c|c|}
\hline$q$ & - & + & & + & & $m$ & & & & & & & & & & $N$ & - & & + & & & & \\
\hline F & - & - & & & & $n$ & $\sim$ & & & & & & & & & $N$ & - & & & & - & & \\
\hline$F$ & + & - & & & & in & & & & & & & & & & - & + & + & & & - & & \\
\hline F & - & - & & & & in & + & & & & & & & & & + & + & + & & & & & \\
\hline ले & - & - & & & & + & - & & & & & & & & & - & & & & & & & \\
\hline$\underset{ల}{\infty}$ & + & - & & & & $\theta$ & - & & & & & & & & & + & + & & & - & + & & \\
\hline m & - & & + & & & $\nabla$ & $\sim$ & & & & & & & & & + & + & & & & & & \\
\hline ల & + & + & + & & & $\nabla$ & $\sim$ & & + & & & & + & & & $N$ & - & + & & & & & \\
\hline$\Leftrightarrow$ & - & - & & & & $n$ & + & & & & & & - & & & - & - & & & + & & & \\
\hline "ా & + & & & & & $n$ & $N$ & & & + & & & + & & & N & & & & & & - & \\
\hline$m$ & - & + & & & & + & $N$ & & & & & & & & & - & + & & & & & & \\
\hline లు & - & - & - & & & $n$ & $\sim$ & & & & & & & & & - & - & & & & & - & \\
\hline $\bar{m}$ & - & + & & & & in & + & + & & & & & & & & & - & & & & & & \\
\hline లి & $N$ & - & & & & + & & & & & & & - & & & + & & + & & & & & \\
\hline సิ & - & - & & & & $\nabla$ & - & & & & & & & & & + & & & + & - & & + & \\
\hline$\stackrel{\sim}{\sim}$ & + & + & & & & $\nabla$ & $\sim$ & & & + & & & + & & & + & $\sim$ & + & & & & & \\
\hline$\hat{\imath}$ & - & - & & - & & $\nabla$ & - & + & & & & & + & & & - & & + & & - & + & & \\
\hline$\stackrel{i}{i}$ & - & & & & & $\nabla$ & & & & & & & & & 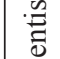 & & + & & & & + & + & \\
\hline $\mathscr{A}$ & + & + & & + & $\begin{array}{l}\stackrel{0}{0} \\
\Xi\end{array}$ & $\nabla$ & - & & & & & + & & & 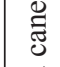 & - & - & + & & - & & & \\
\hline$\underset{d}{ }$ & + & + & & + & $\begin{array}{l}0 \\
\dot{0} \\
0\end{array}$ & $n$ & - & + & & & & + & & & 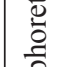 & $\sim$ & - & + & & - & & + & \\
\hline$\pi$ & + & + & + & & $\overrightarrow{0}$ & $\nabla$ & - & + & & & & & & & त्र & & - & + & & - & & + & \\
\hline 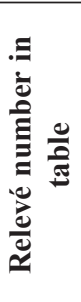 & 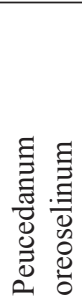 & 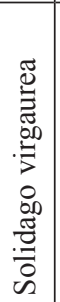 & 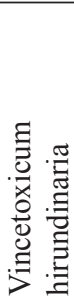 & 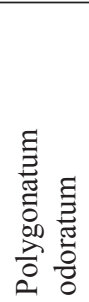 & 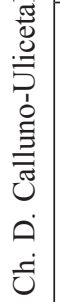 & 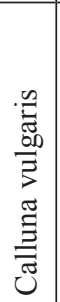 & 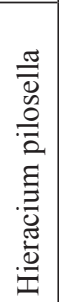 & 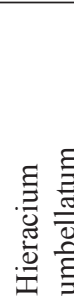 & 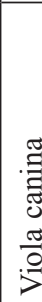 & 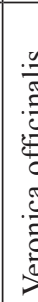 & & 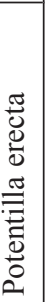 & 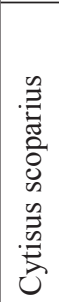 & 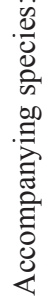 & 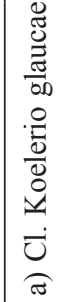 & 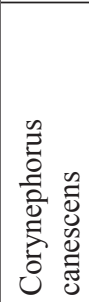 & 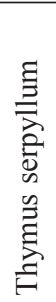 & 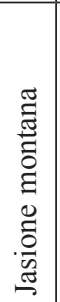 & 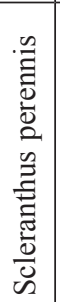 & 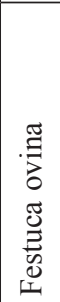 & 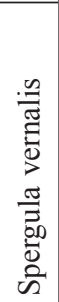 & 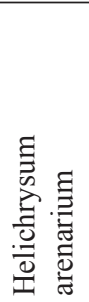 & 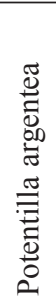 \\
\hline
\end{tabular}




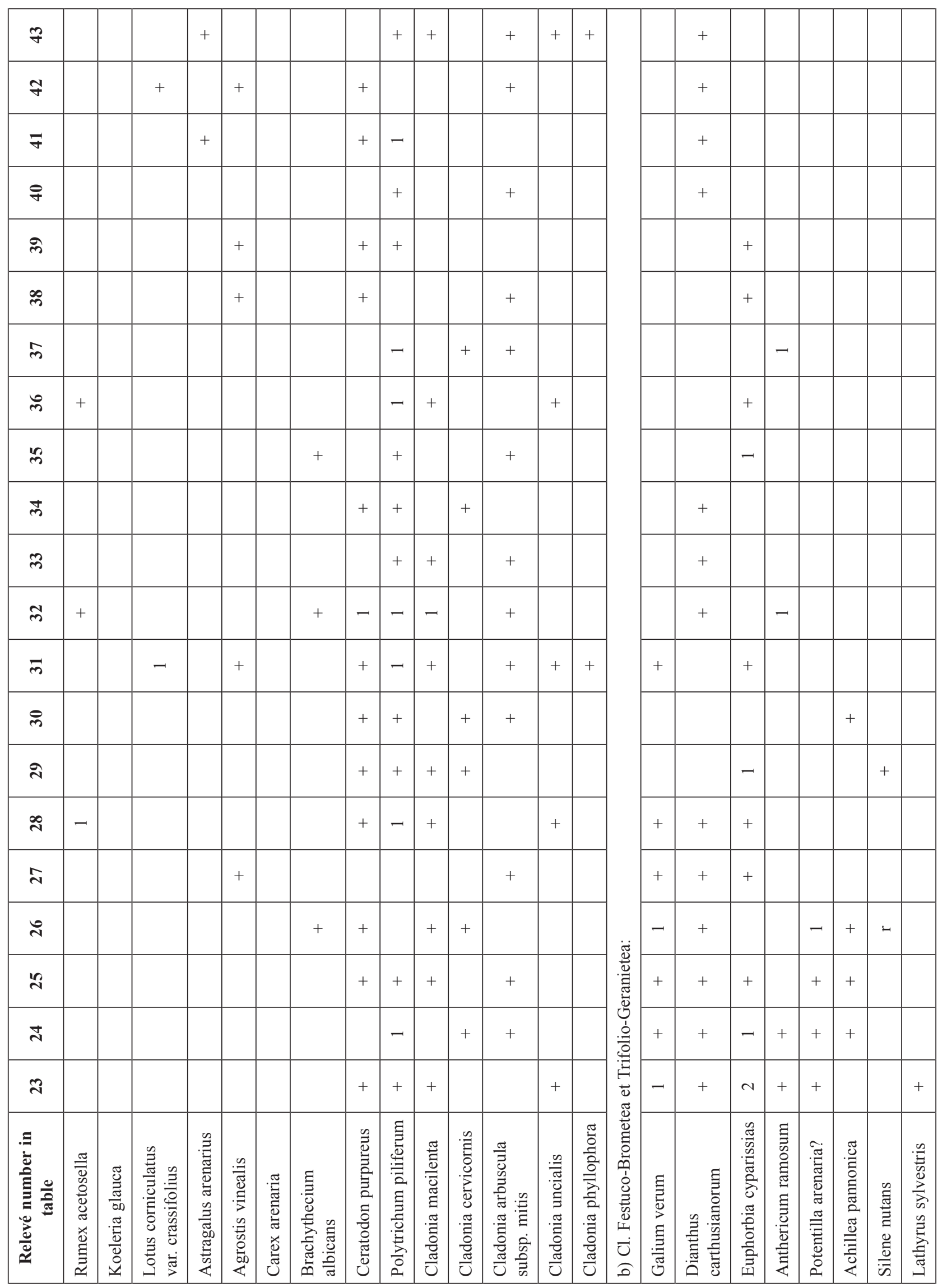




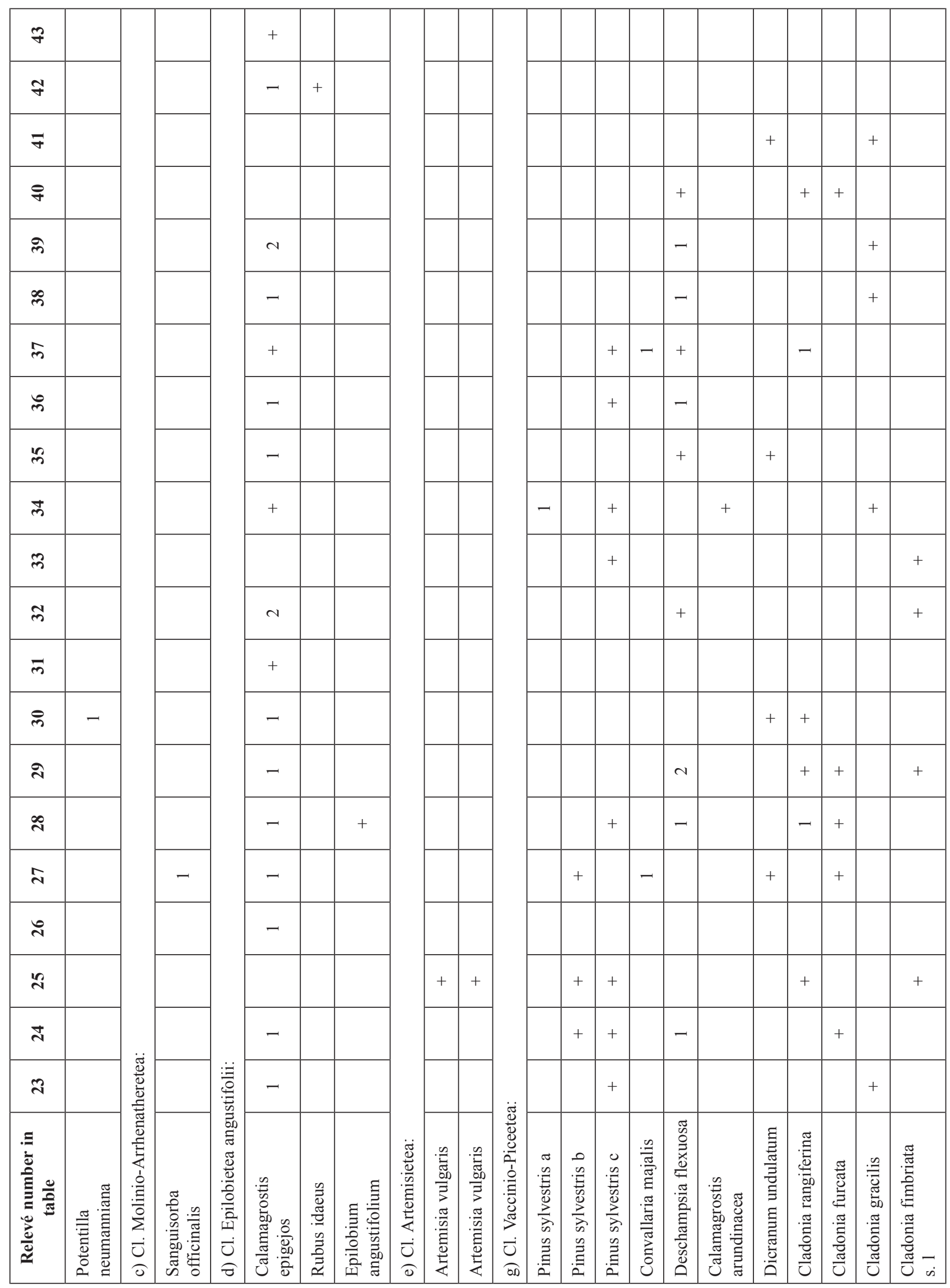




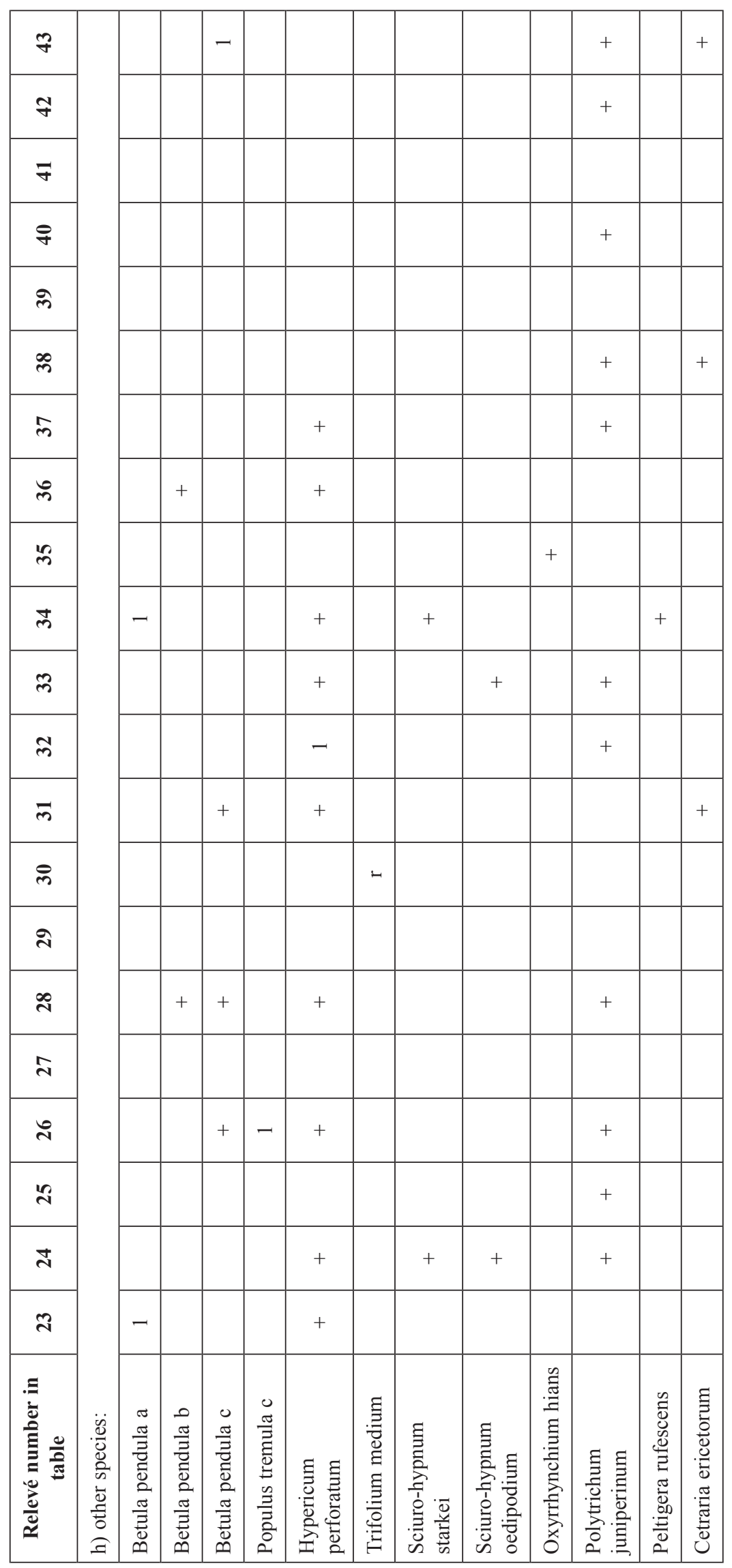




\begin{tabular}{|c|c|c|c|c|c|c|c|c|c|c|c|c|c|c|c|c|c|c|c|c|c|}
\hline 8 & $\hat{\imath}$ & त் & $\bar{m}$ & $\stackrel{x}{0} \circ$ & & & & & 8 & 은 & $\simeq$ & & & & + & & & & & - & - \\
\hline 8 & $\vec{\sim}$ & $\stackrel{\infty}{\infty}$ & $\stackrel{\infty}{m}$ & $\stackrel{\star x}{0} \circ$ & & & & & \& & $\stackrel{0}{=}$ & 2 & & + & & + & + & & & & - & + \\
\hline t & $\stackrel{\vartheta}{\Xi}$ & $\stackrel{\circ}{\circ}$ & $\stackrel{\circ}{\sim}$ & 류 & & & & & \& & ¿ & $\because$ & & + & & + & + & - & & & - & + \\
\hline 3 & $\cong$ & $\stackrel{\infty}{\rightarrow} \stackrel{\infty}{\circ}$ & 워 & 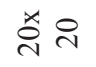 & 은 & 띠 & & & \& & 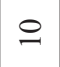 & $\because$ & & & & + & & & & & - & - \\
\hline ช్ & $\stackrel{\cong}{=}$ & $\stackrel{\infty}{\rightarrow} \stackrel{\infty}{\circ}$ & 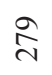 & ڤ્ণ & 으 & z & in & in & $\infty$ & i & ते & & & & & & & & & + & + \\
\hline 5 & $\hat{\varrho}$ & $\stackrel{\infty}{\sim} \stackrel{\infty}{\circ}$ & $\stackrel{?}{\sim}$ & 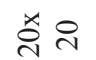 & ते & 学 & & & \& & 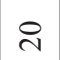 & $\eta$ & & + & & + & + & & & & + & - \\
\hline 8 & $\bar{\sigma}$ & $\therefore$ 残 & ते & 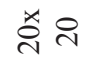 & & & & 으 & $\triangleright$ & $\circ$ & $\approx$ & & + & & + & + & - & & & & + \\
\hline in & \& & $\therefore$ 웅 & ते & ڤ્సે & & & & & \& & ㄱ. & $\vec{N}$ & & + & & + & + & & & & - & + \\
\hline in & $\stackrel{2}{2}$ & $\therefore$ 응 & $\stackrel{\sim}{\sim}$ & $\stackrel{\star b}{0} \circ$ & 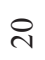 & 3 & in & & \& & $\stackrel{0}{=}$ & \pm & & & + & + & & & & & & + \\
\hline in & $\approx$ & $\therefore 5$ & 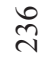 & $\stackrel{\text { ¿ }}{\text { ¿ }}$ & i & z & in & in & 8 & 우 & $=$ & & & & + & & & & & - & + \\
\hline in & $\stackrel{n}{ }$ & $\therefore$ 응 & 品 & $\stackrel{\star}{\sim}$ & i & z & & & \& & 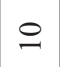 & $\stackrel{\infty}{\sim}$ & & - & + & & & - & & & - & + \\
\hline in & $F$ & $\stackrel{\circ}{0}$ & $\stackrel{\infty}{\sim}$ & $\stackrel{\star}{\circ} \circ$ & & & in & & \& & 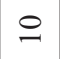 & $\ddot{\lambda}$ & & & + & + & & + & & & + & + \\
\hline in & 8 & $\stackrel{\circ}{0}$ & $\stackrel{\sim}{\sim}$ & $\stackrel{\leftarrow}{0} \simeq$ & శి & z & in & in & \& & $\varrho$ & 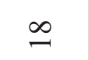 & & & + & + & & + & & & - & + \\
\hline in & $\widetilde{\sigma}$ & $\stackrel{\dot{0}}{0}$ & $\stackrel{\sim}{\sim}$ & $\underset{\sim}{\stackrel{*}{i}}$ & in & z & in & in & $\&$ & 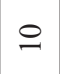 & ¿ิ & & + & & + & + & + & & & + & + \\
\hline हี & $\tilde{n}$ & $\stackrel{\circ}{0}$ & $\underset{\sim}{\sim}$ & 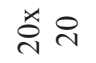 & & & in & & $\infty$ & خి & $\stackrel{0}{0}$ & & & & + & & + & & & + & - \\
\hline $\bar{n}$ & f & $\stackrel{\hat{a}}{r}$ & $\stackrel{n}{\sim}$ & 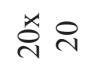 & & & in & in & \& & 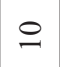 & 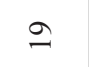 & & & & + & & & & & + & - \\
\hline in & $\vec{\gamma}$ & $\stackrel{0}{i}$ & $\hat{\sigma}$ & ڤ્సે ন & & & & & \& & ㄱ. & $\vec{N}$ & & - & & + & + & & & & & + \\
\hline$\vec{q}$ & के & i் & $\stackrel{\overbrace{}}{\sim}$ & ্ֻণ & 은 & z & & 으 & $\infty$ & 은 & $\vec{N}$ & & & & & & & - & $\ddot{0}$ & - & - \\
\hline$\stackrel{\infty}{+}$ & $\dot{m}$ & तं & के & $\stackrel{\not}{\circ} \circ$ & ㄱ. & $\infty$ & & ণ & $\infty$ & ๙ิ & $\vec{\sim}$ & & + & & + & & & & 렬 & - & - \\
\hline F & $\vec{m}$ & त் & के & 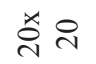 & $\circ$ & z & & 으 & $\triangleright$ & 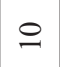 & $\stackrel{d}{J}$ & & & & + & & & - & 总 & $\sim$ & - \\
\hline$q$ & $\simeq$ & $\stackrel{\infty}{\infty}$ & $\stackrel{\infty}{n}$ & 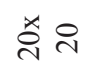 & & & & & $\triangleright$ & ণి & 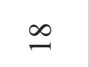 & 美 & & & & & & & 严 & - & - \\
\hline f & $a$ & \begin{tabular}{l}
8 \\
\hdashline
\end{tabular} & $\frac{0}{m}$ & $\stackrel{x}{0} \circ$ & & & & & 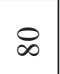 & i & $\infty$ & $\stackrel{1}{ٍ}$ & + & & + & & & & $\begin{array}{l}\overrightarrow{0} \\
\Xi\end{array}$ & - & - \\
\hline 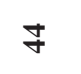 & $r$ & $\stackrel{8}{\circ}$ & $\stackrel{n}{m}$ & 호 & ஜิ & z & & & 8 & in & $\stackrel{ \pm}{\mathrm{N}}$ & $\overrightarrow{0}$ & + & & + & + & & & 苞 & - & \\
\hline 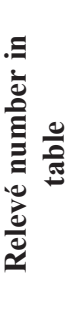 & 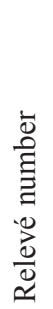 & $\frac{\mathscr{\Xi}}{\tilde{\Xi}}$ & 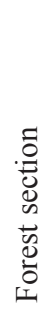 & 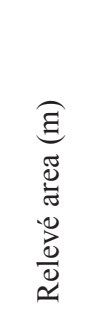 & $\begin{array}{l}\sigma \\
o \\
\frac{0}{0} \\
\text { n. }\end{array}$ & 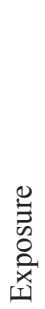 & 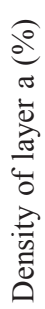 & 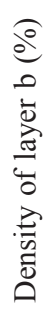 & 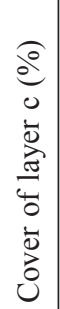 & 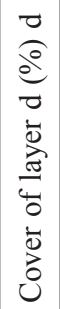 & 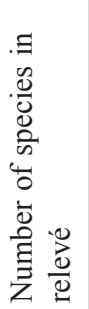 & 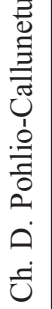 & 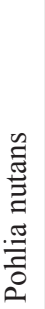 & 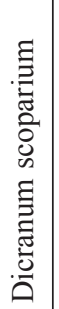 & 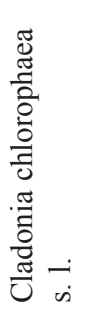 & 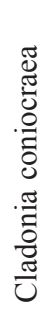 & 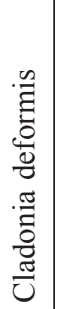 & 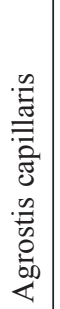 & 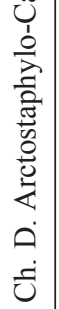 & 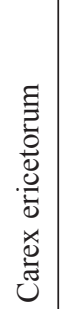 & 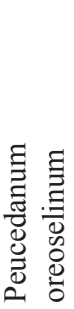 \\
\hline
\end{tabular}




\begin{tabular}{|c|c|c|c|c|c|c|c|c|c|c|c|c|c|c|c|c|c|c|c|c|c|c|c|}
\hline 8 & - & & & & in & - & & & & & & $N$ & & & & & & & & & & & \\
\hline 8 & + & & & & $\nabla$ & & & & + & & & & + & & & & + & & & & + & & \\
\hline t & - & & & & ナ & N & & & & & & & & + & & & & & & + & & & \\
\hline 3 & - & & & & in & - & & & & & & & + & & & & & & & & & & \\
\hline$\widetilde{\sigma}$ & - & + & + & & $m$ & - & & + & & & & - & + & & & & & & & & & & \\
\hline$\overline{6}$ & - & + & & & $\nabla$ & $N$ & & & & & & $m$ & + & & & & & & & & & & \\
\hline 8 & + & & & & $m$ & - & & & & & & - & & + & & & & & & & & & \\
\hline in & + & & & & 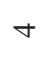 & - & + & & & & & - & - & & + & & & & & & & & \\
\hline in & + & & & & ナ & + & & & & & & - & & & & & & & & & & & \\
\hline in & + & & & & $\nabla$ & + & & & & & & $N$ & & & & & & & & & & & \\
\hline in & + & & & & $\nabla$ & - & + & & & & & - & & + & & - & & & & & & & \\
\hline in & + & & + & & $m$ & + & & & & & & - & & & + & & & & & & & & \\
\hline in & + & & & & $m$ & + & + & & & & & - & & & & & & & & + & & & \\
\hline 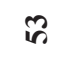 & + & & & & $\nabla$ & & & & & & & + & + & & & & & & & & & & \\
\hline ผี & + & & & & $m$ & & & & & & & $N$ & & & & & & & & & & & \\
\hline $\bar{n}$ & + & & & & $\nabla$ & - & & & & & & - & & & & & & & & & & + & + \\
\hline in & - & & & & $\nabla$ & $n$ & & & & & & $\sim$ & & + & & - & & + & & & & & \\
\hline 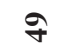 & - & & & & 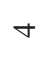 & - & & & & & & $v$ & & & & & + & & & - & & & + \\
\hline$\stackrel{\infty}{+}$ & & & & & $N$ & $\sim$ & + & & & & & $N$ & + & + & & & + & & & & & & \\
\hline f & - & & & & 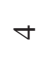 & $\sim$ & - & & - & & : & $\sim$ & & - & & - & & & & & - & & + \\
\hline to & $\sim$ & & & 忢 & 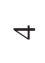 & + & - & & & & $\underset{\widetilde{్}}{\tilde{ల}}$ & + & + & - & + & & & & & & & & \\
\hline 8 & - & & & o & 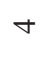 & $\sim$ & & & - & & : & $\sim$ & & & & & & & & + & & & \\
\hline 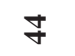 & $n$ & & & ${ }_{\tilde{\omega}}^{Z}$ & $\nabla$ & $N$ & + & & - & & ह્ㅇㅇ & $v$ & & + & & & - & & & & & & \\
\hline 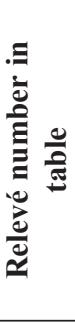 & 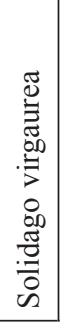 & 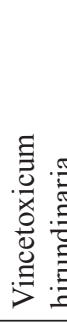 & 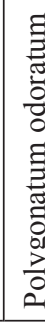 & 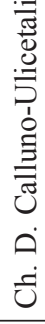 & 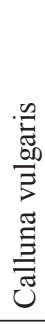 & 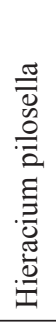 & 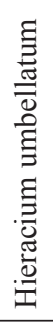 & 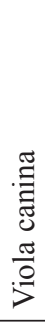 & 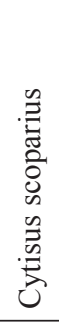 & 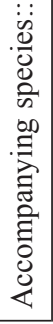 & 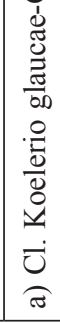 & 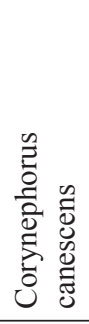 & 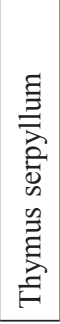 & 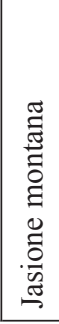 & 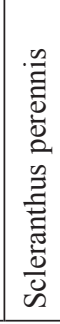 & 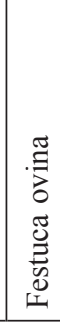 & 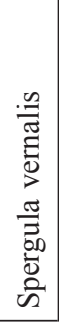 & 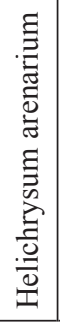 & 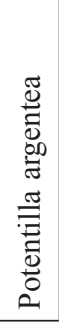 & 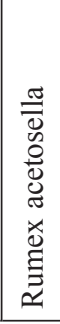 & 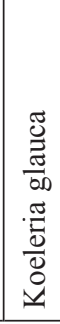 & 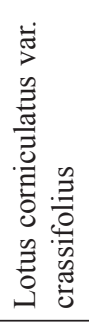 & 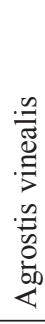 \\
\hline
\end{tabular}




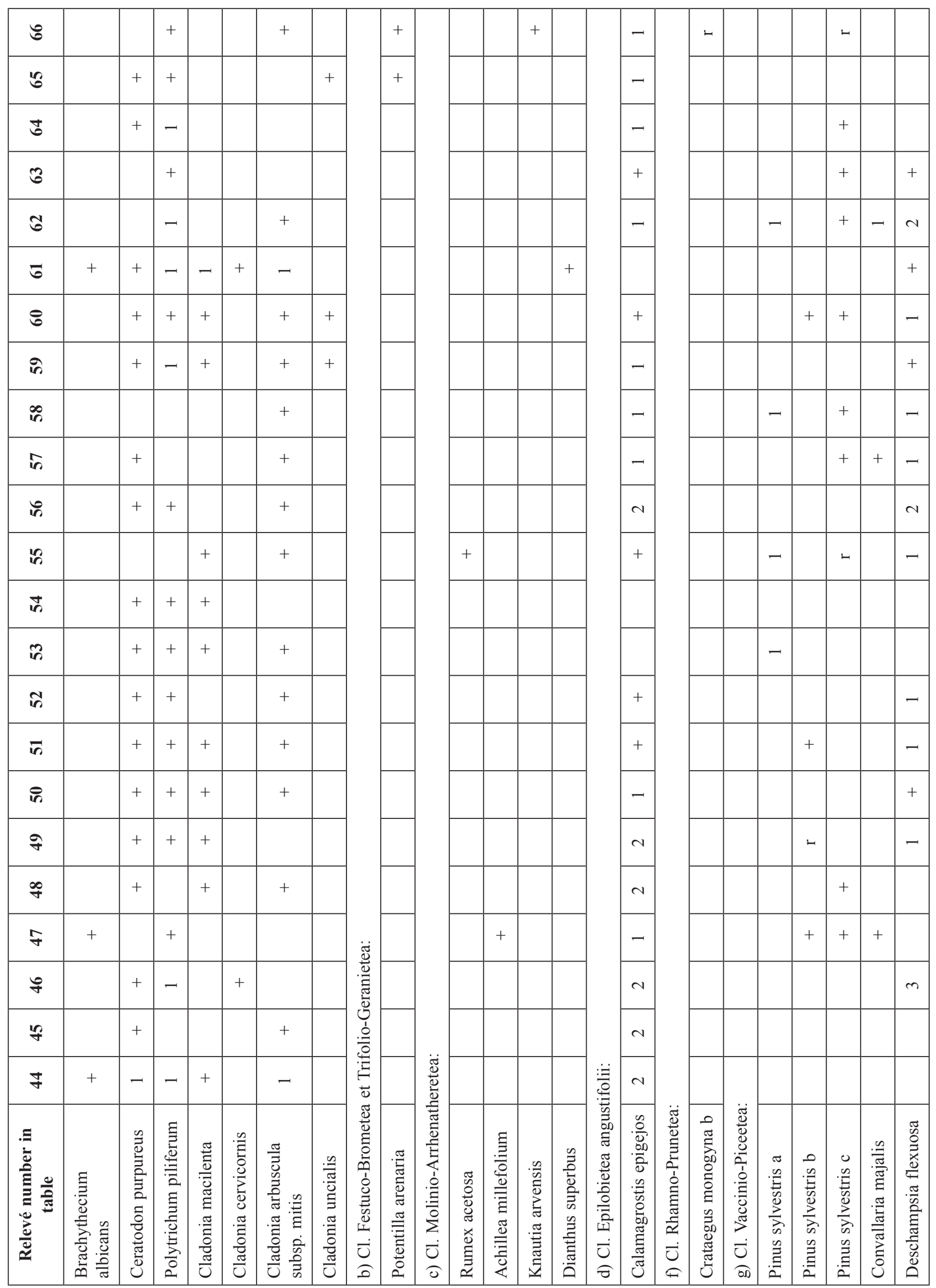




\begin{tabular}{|c|c|c|c|c|c|c|c|c|c|c|c|c|c|c|c|c|c|c|c|c|c|c|c|c|}
\hline 8 & & & & + & & & + & & & & & & & & & & & & & & & & + & \\
\hline 18 & & & & & & & + & + & + & & & & & & & & & & & & & & & \\
\hline$\vec{b}$ & & & & & & & & & & & & & & & & & & & & & & & & \\
\hline 8 & & & & + & + & & + & & & & & & & & & & & & & & & & + & \\
\hline$\widetilde{\sigma}$ & - & & + & & + & + & + & & & & & + & + & & & & & & & + & + & + & + & + \\
\hline 5 & & & & & & & + & + & & & & & & & & & + & & & & & & + & \\
\hline 8 & & & & + & & & & & & & & & $\mathrm{N}$ & & & - & & & & & & & & \\
\hline in & & & & & + & & + & & & & & & & & & & & & & & & & & \\
\hline$\stackrel{\infty}{\infty}$ & & & & + & & & & & & & & & - & & & & & & & & & & & \\
\hline in & & + & & & & & & & & & + & + & + & & & & & & & & & & & \\
\hline in & & & & & & & + & & & & & & & & & & & & & & & & & \\
\hline in & & & & + & & & + & & & & + & & & & & & & & + & & & & + & \\
\hline in & & & & & & & + & & & & - & + & . & & & & & & & & & & + & \\
\hline in & & & & & + & & & & & & & + & + & & & & + & & & & & & + & \\
\hline กี & & & & & + & & + & & & & - & & & & & & & & & & & & + & \\
\hline $\bar{n}$ & & & & & & & & & & & - & + & & & & & & & & & & & + & \\
\hline in & & & & + & & + & & & & & & & + & & & & & & & & & & + & \\
\hline qे & & & & + & & & + & & & & & + & & & & & & & - & & & & + & \\
\hline$\stackrel{\infty}{+}$ & & & & & & & & + & & & & $H$ & & & + & - & & & & & & & + & \\
\hline f & & & + & & & & & & & & & & & + & & & & & & & & & + & \\
\hline fo & & & & + & & & + & & & & & & & & & & & & & & & & & + \\
\hline 8 & & & + & & & & & & & & & & - & & & & & & & + & + & & + & \\
\hline 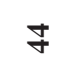 & & & & & & & & & & & & & & & & & & + & & + & + & + & + & + \\
\hline 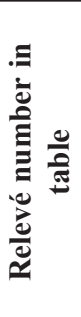 & 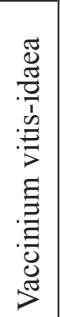 & 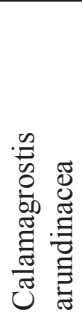 & 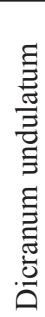 & 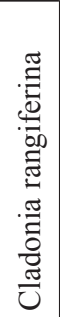 & 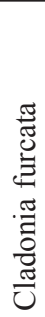 & 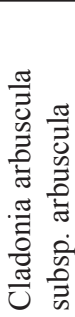 & 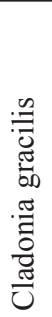 & 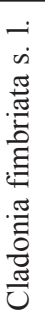 & 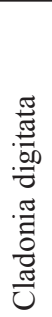 & 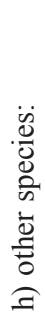 & 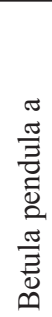 & 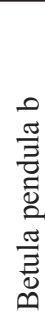 & 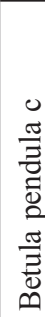 & 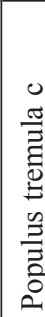 & 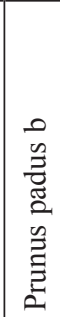 & 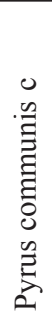 & 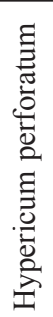 & 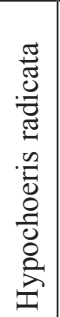 & 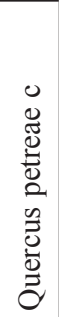 & 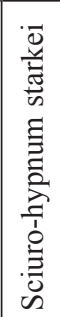 & 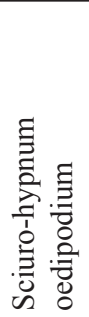 & 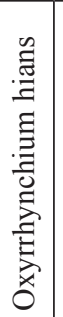 & 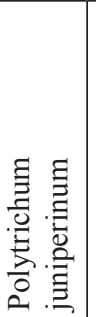 & 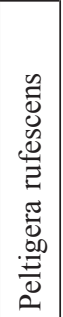 \\
\hline
\end{tabular}


Table 1 (the fifth part). Association Pohlio-Callunetum (relevé 44-73 the typical variant, relevé 67-73 the form with Pleurozium schreberi)

\begin{tabular}{|c|c|c|c|c|c|c|c|}
\hline Relevé number in table & 67 & 68 & 69 & 70 & 71 & 72 & 73 \\
\hline Relevé number & 38 & 40 & 42 & 44 & 55 & 70 & 121 \\
\hline Date & $\begin{array}{c}25.06 \\
2008\end{array}$ & $\begin{array}{l}7.07 . \\
2008\end{array}$ & $\begin{array}{l}7.07 . \\
2008\end{array}$ & $\begin{array}{l}7.07 . \\
2008\end{array}$ & $\begin{array}{c}16.07 \\
2008\end{array}$ & $\begin{array}{c}16.07 \\
2008\end{array}$ & $\begin{array}{c}10.10 \\
2008\end{array}$ \\
\hline Forest section & 276 & 237 & 237 & 275 & 274 & 238 & 240 \\
\hline Relevé area (m) & $20 \times 20$ & $10 \times 10$ & $20 \times 20$ & $10 \times 10$ & $20 \times 20$ & $20 \times 20$ & $20 \times 20$ \\
\hline Slope $\left({ }^{\circ}\right)$ & 10 & & 10 & & & & \\
\hline Exposure & $\mathrm{N}$ & & $\mathrm{NE}$ & & & & \\
\hline Density of layer a (\%) & & 5 & & 5 & & & 5 \\
\hline Density of layer b (\%) & 5 & & & 5 & & & \\
\hline Cover of layer c (\%) & 90 & 90 & 90 & 80 & 90 & 90 & 90 \\
\hline Cover of layer d (\%) & 10 & 20 & 20 & 20 & 10 & 20 & 20 \\
\hline Number of species in relevé & 18 & 22 & 18 & 18 & 25 & 17 & 21 \\
\hline \multicolumn{8}{|c|}{ Ch. D. Pohlio-Callunetum et Pohlio-Callunion } \\
\hline Pohlia nutans & & & + & + & & & 1 \\
\hline Dicranum scoparium & + & + & + & + & & + & + \\
\hline Cladonia chlorophaea & + & + & & + & & + & + \\
\hline Cladonia coniocraea D & & & & & & & + \\
\hline Cladonia deformis & & & & & + & + & \\
\hline Agrostis capillaris & + & & & & & & \\
\hline \multicolumn{8}{|c|}{ Ch. D. Arctostaphylo-Callunetum et Calluno-Arctostaphylion: } \\
\hline Carex ericetorum & + & & & & 1 & + & + \\
\hline Peucedanum oreoselinum & + & & + & 1 & 1 & + & 1 \\
\hline Solidago virgaurea & & 1 & 1 & & + & 1 & + \\
\hline \multicolumn{8}{|l|}{ Vincetoxicum hirundinaria D } \\
\hline \multicolumn{8}{|l|}{ Polygonatum odoratum } \\
\hline \multicolumn{8}{|c|}{ Ch. D. Calluno-Ulicetalia et Nardo-Callunetea: } \\
\hline Calluna vulgaris & 4 & 4 & 5 & 3 & 5 & 4 & 4 \\
\hline Hieracium pilosella & & + & + & & + & 1 & \\
\hline Hieracium umbellatum & & + & & & + & & \\
\hline Viola canina & & & & & + & & \\
\hline \multicolumn{8}{|l|}{ Veronica officinalis } \\
\hline \multicolumn{8}{|l|}{ Potentilla erecta } \\
\hline \multicolumn{8}{|l|}{ Cytisus scoparius } \\
\hline \multicolumn{8}{|l|}{ Accompanying species: } \\
\hline \multicolumn{8}{|c|}{ a) Cl. Koelerio glaucae-Corynephoretea canescentis } \\
\hline Corynephorus canescens & 1 & + & + & & & + & \\
\hline Thymus serpyllum & & & & & 1 & + & \\
\hline Jasione montana & & + & & & & & \\
\hline
\end{tabular}




\begin{tabular}{|c|c|c|c|c|c|c|c|}
\hline Relevé number in table & 67 & 68 & 69 & 70 & 71 & 72 & 73 \\
\hline Scleranthus perennis & & 1 & & & & & \\
\hline Festuca ovina & & 1 & & & & & \\
\hline \multicolumn{8}{|l|}{ Spergula vernalis } \\
\hline \multicolumn{8}{|l|}{ Helichrysum arenarium } \\
\hline \multicolumn{8}{|l|}{ Potentilla argentea } \\
\hline Rumex acetosella & & & & & & & + \\
\hline Koeleria glauca & & & & & + & & \\
\hline \multicolumn{8}{|l|}{ Lotus corniculatus var. crassifolius } \\
\hline \multicolumn{8}{|l|}{ Astragalus arenarius } \\
\hline \multicolumn{8}{|l|}{ Agrostis vinealis } \\
\hline Carex arenaria & & & & & & & 2 \\
\hline Brachythecium albicans & & + & & & & + & + \\
\hline Ceratodon purpureus & + & & + & & & + & \\
\hline Polytrichum piliferum & & & + & & & & + \\
\hline Cladonia macilenta & & & + & + & + & + & \\
\hline \multicolumn{8}{|l|}{ Cladonia cervicornis } \\
\hline Cladina mitis & + & + & & & + & + & \\
\hline Cladonia uncialis & & & & & + & & \\
\hline Cladonia phyllophora & & & & & + & & \\
\hline \multicolumn{8}{|c|}{ b) Cl. Festuco-Brometea et Trifolio-Geranietea: } \\
\hline \multicolumn{8}{|l|}{ Galium verum } \\
\hline \multicolumn{8}{|l|}{ Dianthus carthusianorum } \\
\hline \multicolumn{8}{|l|}{ Euphorbia cyparissias } \\
\hline \multicolumn{8}{|l|}{ Anthericum ramosum } \\
\hline \multicolumn{8}{|l|}{ Potentilla arenaria } \\
\hline \multicolumn{8}{|l|}{ Achillea pannonica } \\
\hline \multicolumn{8}{|l|}{ Silene nutans } \\
\hline \multicolumn{8}{|l|}{ Lathyrus sylvestris } \\
\hline \multicolumn{8}{|l|}{ Potentilla neumanniana } \\
\hline \multicolumn{8}{|l|}{ Geranium sanguineum } \\
\hline \multicolumn{8}{|l|}{ Carlina vulgaris } \\
\hline \multicolumn{8}{|l|}{ c) Cl. Molinio-Arrhenatheretea: } \\
\hline Rumex acetosa & & & & & & & + \\
\hline \multicolumn{8}{|l|}{ Achillea millefolium } \\
\hline \multicolumn{8}{|l|}{ Molinia caerulea } \\
\hline \multicolumn{8}{|l|}{ Knautia arvensis } \\
\hline \multicolumn{8}{|l|}{ Sanguisorba officinalis } \\
\hline \multicolumn{8}{|l|}{ Dianthus superbus } \\
\hline d) Cl. Epilobietea angustifolii: & & & & & & & \\
\hline Calamagrostis epigejos & 1 & 1 & & & + & & \\
\hline
\end{tabular}




\begin{tabular}{|c|c|c|c|c|c|c|c|}
\hline Relevé number in table & 67 & 68 & 69 & 70 & 71 & 72 & 73 \\
\hline \multicolumn{8}{|l|}{ Rubus idaeus } \\
\hline \multicolumn{8}{|l|}{ Epilobium angustifolium } \\
\hline \multicolumn{8}{|l|}{ e) Cl. Artemisietea: } \\
\hline \multicolumn{8}{|l|}{ Artemisia vulgaris } \\
\hline \multicolumn{8}{|l|}{ Linaria vulgaris } \\
\hline \multicolumn{8}{|l|}{ Verbascum densiflorum } \\
\hline \multicolumn{8}{|l|}{ f) Cl. Rhamno-Prunetea: } \\
\hline \multicolumn{8}{|l|}{ Crataegus monogyna b } \\
\hline \multicolumn{8}{|l|}{ Berberis vulgaris $b$} \\
\hline \multicolumn{8}{|l|}{ g) Cl. Vaccinio-Piceetea: } \\
\hline Pinus sylvestris a & & & & & & & 1 \\
\hline Pinus sylvestris b & + & & & + & & & \\
\hline Pinus sylvestris c & & & & & + & & \\
\hline Convallaria majalis & & & & + & & & \\
\hline Deschampsia flexuosa & 2 & 2 & + & 2 & & & + \\
\hline Vaccinium vitis-idaea & & & & 2 & & & \\
\hline \multicolumn{8}{|l|}{ Vaccinium myrtillus } \\
\hline \multicolumn{8}{|l|}{ Calamagrostis arundinacea } \\
\hline Pleurozium schreberi & 1 & 2 & 1 & 2 & 2 & 1 & 2 \\
\hline Dicranum polysetum & + & + & + & + & + & + & + \\
\hline Cladonia rangiferina & & & & + & + & & + \\
\hline Cladonia furcata & & & & & & & + \\
\hline Cladonia arbuscula & + & + & & & & & \\
\hline Cladonia gracilis & & & + & + & & & \\
\hline Cladonia fimbriata & & & + & & & & \\
\hline Cladonia squamosa & + & + & + & + & + & & + \\
\hline Cladonia digitata & & & & & + & & \\
\hline \multicolumn{8}{|l|}{ h) other species: } \\
\hline Betula pendula a & & 1 & & 1 & & & \\
\hline Betula pendula $\mathrm{b}$ & + & & & & & & \\
\hline Betula pendula c & & & & 2 & & + & \\
\hline \multicolumn{8}{|l|}{ Populus tremula b } \\
\hline Populus tremula c & & & & & 1 & & \\
\hline Hypericum perforatum & & & & & + & & \\
\hline Senecio vulgaris & & + & & & & & \\
\hline Sciuro-hypnum oedipodium & + & & + & & & & + \\
\hline \multicolumn{8}{|l|}{ Oxyrrhynchium hians } \\
\hline \multicolumn{8}{|l|}{ Polytrichum juniperinum } \\
\hline Peltigera rufescens & & + & + & + & + & & \\
\hline Cetraria ericetorum & & & & & + & & \\
\hline
\end{tabular}


tinguished by the presence of species from the classes Festuco-Brometea and Trifolio-Geranietea, such as Galium verum, Dianthus carthusianorum, Euphorbia cyparissias, Anthericum ramosum. One can distinguish two subvariants within this variant: typical and the one with purple moor grass Molinia caerulea. The latter develops mainly in depressions between dunes, where soil moisture is periodically higher. Therefore, it is characterized by higher species richness. Apart from characteristic and differential species of the association Pohlio-Callunetum, numerous species of mosses and lichens are present in its patches, forming a relatively well developed layer of mosses and lichens, covering on average $20 \%$ of the relevé's area. In the layer of herbaceous vegetation, apart from purple moor grass Molinia caerulea, usually also chee reedgrass Calamagrostis epigejos occurs. Whereas, among the most frequent mosses and lichens of the described community, the following are included: Pohlia nutans, Dicranum scoparium, Polytrichum piliferum, Ceratodon purpureus.

Additionally, in patches of the typical subvariant, one can distinguish a form with Pleurozium schreberi, which develops in places adjacent to pine forests. Its species composition is characterized by the presence of many species from the class Vaccinio-Piceetea, such as: the grass Deschampsia flexuosa, the moss Dicranum undulatum and lichens Cladonia gracilis and C. arbuscula subsp. arbuscula.

\subsubsection{Association Arctostaphylo-Callunetum}

Phytocoenoses of the bearberry heath Arctostaphylo-Callunetum cover a much smaller area in the studied area. They develop in the form of two variants: typical and the one with wild thyme Thymus serpyllum (Tab. 2).

The typical variant usually covers insolated sites on the tops of dunes and hills, less frequently on slopes of small inclination and southern exposure. Arctostaphylos uva-ur$s i$ definitely dominates in its patches. Clusters of heather are small and very sparse. Therefore on the exposed soil beneath the heather, heliophilous species of lichens may settle, mainly Cladonia arbuscula subsp. mitis, C. furcata, C. cervicornis and C. chlorophaea s. 1. Also, the high abundance of kinnikinnick bearberry is related to the type of habitat, which is open and exposed to strong insolation. In the patches of the described community, besides bryophytes and lichens, often other short plants grow, e.g. Hieracium pilosella. Also seedlings of trees and shrubs appear individually and dispersed, mainly Scots pine Pinus sylvestris and European white birch Betula pendula. Depending on the light conditions, the cover of the herbaceous layer reaches $80-90 \%$, whereas the moss layer covers from 5 to $20 \%$ (Tab. 2).

The variant with Thymus serpyllum occurs most frequently on sunny and warm exposures, but not on the tops of hills and other elevations. More frequently one can also find it on flat areas or on slopes with small inclination. Its patches are characterized by the domination of Calluna vulgaris, which often forms facies, reaching the maximum cover. The heather, through limiting the access of light to the substrate, causes that the bearberry, as well as mosses and lichens from the class Koelerio glaucaeCorynephoretea canescentis occur in smaller quantities.

As compared with the typical variant, the variant with Thymus serpyllum is characterized by a higher contribution of species differential for the association ArctostaphyloCallunetum and the alliance Calluno-Arctostaphylion, such as: Carex ericetorum, Peucedanum oreoselinum and Solidago virgaurea. Thymus serpyllum occurs in all patches of the variant. Moreover, those patches are characterized by a higher number of species, ranging from 23 to 36 . At the same time, their floristic composition comprises lots of species of sandy grasslands from the class Koelerio glaucae-Corynephoretea canescentis, such as the aforementioned Thymus serpyllum, or Corynephorus canescens and Potentilla argentea. Whereas from among heath plants, Hieracium pilosella occurs, as well as occasionally Viola canina, Veronica officinalis and Potentilla erecta. Moreover, the variant with Thymus serpyllum is characterized by higher contribution of species from the class Vaccinio-Piceetea such as: Deschampsia flexuosa and Pinus sylvestris, and also lichens and mosses: Cladonia arbuscula subsp. arbuscula, C. fimbriata s. 1., C. rangiferina, Dicranum undulatum, Pleurozium schreberi. In the patches of this variant, also species from the class Festuco-Brometea and Trifolio-Geranietea grow: Potentilla arenaria, Hypericum perforatum, Galium verum, Dianthus carthusianorum, Euphorbia cyparissias, Anthericum ramosum, Achillea pannonica and Filipendula vulgaris (reaching the degree of constancy II or I, Tab. 2).

\subsubsection{The community with Cytisus scoparius}

The community dominated by common groom- Cytisus scoparius covers the areas adjacent to heathlands PohlioCallunetum and Arctostaphylo-Callunetum, as well as with grasslands of the gray clubawn grass Spergulo vernalisCorynephoretum. Based on the analysis of 8 relevés from the training ground, two forms of the described community were distinguished: the typical one and the form with $D i$ anthus carthusianorum (Tab. 3).

The typical form covers gentle slopes of northern or southern exposure. Apart from the domination of common broom, it is characterized by the significant contribution of heathland species from the class Nardo-Callunetea, such as: Calluna vulgaris and to a lesser extent Carex ericetorum. However, it is necessary to emphasize that due to considerable shadiness in the middle of patches brought on by common groom, the aforementioned species, similarly 
Table 2. The association Arctostaphylo-Callunetum (relevés 1-6, the variant with Thymus serpyllum, relevés 7-11, the typical variant)

\begin{tabular}{|c|c|c|c|c|c|c|c|c|c|c|c|c|}
\hline Relevé number in table & 1 & 2 & 3 & 4 & 5 & 6 & 7 & 8 & 9 & 10 & 11 & Constancy \\
\hline Relevé number & 116 & 94 & 112 & 73 & 111 & 99 & 86 & 87 & 88 & 89 & 98 & \\
\hline Date & $\begin{array}{c}10.09 \\
2008\end{array}$ & $\begin{array}{c}17.07 \\
2008\end{array}$ & $\begin{array}{c}18.08 \\
2008\end{array}$ & $\begin{array}{c}17.07 \\
2008\end{array}$ & $\begin{array}{c}18.08 \\
2008\end{array}$ & $\begin{array}{c}18.08 \\
2008\end{array}$ & $\begin{array}{c}11.10 \\
2008\end{array}$ & $\begin{array}{c}11.10 . \\
2008\end{array}$ & $\begin{array}{c}11.10 \\
2008\end{array}$ & $\begin{array}{c}11.10 \\
2008\end{array}$ & $\begin{array}{c}18.08 \\
2008\end{array}$ & \\
\hline Forest section & 240 & 239 & 279 & 236 & 279 & 279 & 279 & 279 & 279 & 279 & 279 & \\
\hline Relevé area (m) & $20 \times 20$ & $20 \times 20$ & $10 \times 10$ & $10 \times 10$ & $10 \times 10$ & $10 \times 10$ & $10 \times 10$ & $10 \times 10$ & $10 \times 10$ & $10 \times 10$ & $10 \times 10$ & \\
\hline Slope $\left(^{\circ}\right)$ & & 10 & & & 10 & 10 & & 10 & & 10 & & \\
\hline Exposure & & $\mathrm{N}$ & & & S & E & & S & & S & & \\
\hline Density of layer a (\%) & 10 & 20 & 10 & 10 & 5 & 5 & 10 & 10 & 5 & 10 & 10 & \\
\hline Density of layer b (\%) & & 5 & 5 & 10 & 5 & 5 & 5 & 10 & 10 & 5 & 5 & \\
\hline Cover of layer c (\%) & 90 & 90 & 70 & 90 & 70 & 80 & 80 & 80 & 80 & 90 & 80 & \\
\hline Cover of layer d (\%) & 20 & 10 & 20 & 10 & 20 & 10 & 10 & 20 & 10 & 10 & 5 & \\
\hline Number of species in relevé & 38 & 26 & 24 & 27 & 23 & 24 & 18 & 19 & 16 & 16 & 12 & \\
\hline \multicolumn{13}{|c|}{ Ch. D Arctostaphylo-Callunetum et Calluno-Arctostaphylion: } \\
\hline Arctostaphylos uva-ursi & + & + & 2 & 1 & 2 & 1 & 4 & 4 & 5 & 5 & 4 & $\mathrm{~V}$ \\
\hline Solidago virgaurea & 1 & + & 1 & + & + & + & + & + & + & & 1 & $\mathrm{~V}$ \\
\hline Peucedanum oreoselinum & 1 & + & + & + & + & 1 & + & + & + & + & + & V \\
\hline Carex ericetorum & 2 & & + & + & & & + & + & + & + & + & IV \\
\hline Vincetoxicum hirundinaria & + & + & 1 & & + & & & & & & & II \\
\hline Polygonatum odoratum & & & & + & & & & & & & & I \\
\hline \multicolumn{13}{|c|}{ Ch. D. Calluno-Ulicetalia et Nardo-Callunetea: } \\
\hline Calluna vulgaris & 4 & 4 & 3 & 3 & 3 & 4 & 1 & + & + & 1 & 1 & $\mathrm{~V}$ \\
\hline Hieracium pilosella & 2 & + & 2 & & 1 & 1 & 1 & + & & + & & IV \\
\hline Viola canina & 1 & & & & & + & & & & & & I \\
\hline Veronica officinalis & + & & + & & & & & & & & & I \\
\hline Potentilla erecta & + & + & & & & & & & & & & I \\
\hline Hieracium umbellatum & & & + & & & & & & & & & I \\
\hline Pohlia nutans & + & & & & + & + & + & & & & & II \\
\hline Dicranum scoparium & + & & + & & & + & & & & & & II \\
\hline Cladonia chlorophaea s. 1. & & & + & + & + & & + & + & & & + & III \\
\hline Cladonia coniocraea & + & & & 1 & & & & & & + & & II \\
\hline \multicolumn{13}{|l|}{ Accompanying species: } \\
\hline \multicolumn{13}{|c|}{ a) Cl. Koelerio glaucae-Corynephoretea canescentis } \\
\hline Corynephorus canescens & + & 1 & + & 1 & + & & 1 & 1 & + & + & & V \\
\hline Thymus serpyllum & + & + & 1 & + & 1 & + & & + & & & & IV \\
\hline Jasione montana & + & & & & & & & & & & & I \\
\hline Scleranthus perennis & & & & + & & & & & & & & I \\
\hline Potentilla argentea & + & & & + & & & & & & & & I \\
\hline Rumex acetosella & + & & & & & & & & & & & I \\
\hline Koeleria glauca & & & & & & + & & & & & & I \\
\hline
\end{tabular}




\begin{tabular}{|l|c|c|c|c|c|c|c|c|c|c|c|c|}
\hline \multicolumn{1}{|c|}{ Relevé number in table } & $\mathbf{1}$ & $\mathbf{2}$ & $\mathbf{3}$ & $\mathbf{4}$ & $\mathbf{5}$ & $\mathbf{6}$ & $\mathbf{7}$ & $\mathbf{8}$ & $\mathbf{9}$ & $\mathbf{1 0}$ & $\mathbf{1 1}$ & Constancy \\
\hline Carex arenaria & 1 & & & & & & & & & & & I \\
\hline Ceratodon purpureus & + & & & & + & & & + & & & + & II \\
\hline Polytrichum piliferum & & + & & & + & 1 & 1 & 1 & + & + & + & IV \\
\hline Cladonia macilenta & + & & + & & + & + & + & + & + & & + & IV \\
\hline Cladonia cervicornis & & + & & + & & & + & & + & + & & III \\
\hline $\begin{array}{l}\text { Cladonia arbuscula subsp. } \\
\text { mitis }\end{array}$ & + & + & & + & & 1 & & + & & + & & III \\
\hline Cladonia furcata & & & + & & + & & + & & & + & + & III \\
\hline
\end{tabular}

b) Cl. Festuco-Brometea i Trifolio-Geranietea:

\begin{tabular}{|l|c|c|c|c|c|c|c|c|c|c|c|c|}
\hline Potentilla arenaria & + & + & + & + & & & & & & & II \\
\hline Hypericum perforatum & 1 & + & + & & & & & & & & & II \\
\hline Galium verum & + & + & & & & & & & & & & I \\
\hline Dianthus carthusianorum & + & & + & & & & & & & & & I \\
\hline Euphorbia cyparissias & + & + & & & & & & & & & & I \\
\hline Anthericum ramosum & & + & & & & & & & & & & I \\
\hline Achillea pannonica & 1 & & & & & & & & & & & I \\
\hline Filipendula vulgaris & 2 & & & & & & & & & & & I \\
\hline
\end{tabular}

c) Cl. Molinio-Arrhenatheretea:

Rumex acetosa

Achillea millefolium

Molinia caerulea

d) $\mathrm{Cl}$. Epilobietea angustifolii:

\begin{tabular}{|c|c|c|c|c|c|c|c|c|c|c|c|c|}
\hline Calamagrostis epigejos & 2 & 1 & + & + & + & + & & + & & + & & IV \\
\hline \multicolumn{13}{|l|}{ e) Cl. Vaccinio-Piceetea: } \\
\hline Pinus sylvestris a & 2 & 3 & 1 & 2 & 2 & 3 & 1 & 1 & 1 & 1 & 1 & $\mathrm{~V}$ \\
\hline Pinus sylvestris $b$ & + & & + & 1 & + & + & + & 1 & + & + & + & IV \\
\hline Pinus sylvestris c & + & + & & 1 & & + & + & + & + & & & III \\
\hline Deschampsia flexuosa & & & + & 1 & 1 & + & & & & & & II \\
\hline Vaccinium vitis-idaea & & & & 1 & & & & & & & & I \\
\hline Vaccinium myrtillus & & + & & + & & & & & & & & I \\
\hline Pleurozium schreberi & & + & & + & & & & & & & & I \\
\hline Dicranum undulatum & + & + & & + & + & & & & & & & II \\
\hline Cladonia rangiferina & + & & + & & + & + & + & & & & & III \\
\hline Cladonia fimbriata s. 1. & & + & + & & + & + & & & + & & & III \\
\hline $\begin{array}{l}\text { Cladonia arbuscula subsp. } \\
\text { arbuscula }\end{array}$ & + & & & + & & + & & & & & & II \\
\hline \multicolumn{13}{|l|}{ f) other species: } \\
\hline Betula pendula a & & & & & 1 & 1 & & & 1 & 1 & & III \\
\hline Betula pendula $b$ & & & & & & & & + & & & & I \\
\hline Betula pendula c & & + & & & + & & & & + & + & & III \\
\hline Polytrichum juniperinum & + & + & & & & + & + & & + & & & III \\
\hline
\end{tabular}


like lichens and mosses usually cover the sites on the edge of the community.

The form with Dianthus carthusianorum differs from the typical form mainly in the higher abundance of species in the patches. Most commonly it develops in flat places. Its patches are distinguished by the presence of Dianthus carthusianorum, as well as by great constancy of Hypericum perforatum and species from the class Koelerio glaucae-Corynephoretea canescentis, such as: Jasione montana, Scleranthus perennis, Hypohoeris radicata, Helichrysum arenarium and Rumex acetosella. The layer of mosses and lichens is more properly developed here as compared to the typical form. Among others, it is composed by: Pohlia nutans, Dicranum scoparium, Cladonia coniocraea, C. deformis from the class Nardo-Callunetea, as well as Polytrichum piliferum, Cladonia uncialis, C. macilenta, from the class Koelerio glaucae-Corynephoretea canescentis. Also species from the class Vaccinio-Piceetea are present, mainly: Solidago virgaurea and Deschampsia flexuosa, as well as single specimens of Pinus sylvestris and Betula pendula.

\subsection{Communities accompanying the heaths}

The above mentioned and described heath communities occur in the analysed territory as a mosaic with arenaceous grasslands and spontaneously developed pine and birch forests from the class Vaccinio-Piceeta. Among heathlands, also the association Calamagrostietum epigeji is relatively frequently encountered.

\subsubsection{Psammophilous grasslands}

Psammophilous grasslands on the artillery range near Torun are represented by the association Spergulo vernalis-Corynephoretum (Tab. 4). They cover a very large area here. They develop most completely on insolated slopes

Table 3. The community with Cytisus scoparius (relevés 1-4, the typical form; relevés 5-8, the form with Dianthus carthusianorum)

\begin{tabular}{|c|c|c|c|c|c|c|c|c|c|}
\hline Relevé number in table & 1 & 2 & 3 & 4 & 5 & 6 & 7 & 8 & Constancy \\
\hline Relevé number & 14 & 15 & 6 & 20 & 27 & 59 & 118 & 18 & \\
\hline Date & $\begin{array}{c}18.06 \\
2008\end{array}$ & $\begin{array}{c}18.06 \\
2008\end{array}$ & $\begin{array}{c}29.05 . \\
2008\end{array}$ & $\begin{array}{c}18.06 \\
2008\end{array}$ & $\begin{array}{c}22.06 \text {. } \\
2008\end{array}$ & $\begin{array}{c}16.07 \\
2008\end{array}$ & $\begin{array}{c}10.10 \\
2008\end{array}$ & $\begin{array}{c}18.06 . \\
1008\end{array}$ & \\
\hline Forest section & 358 & 358 & 357 & 358 & 359 & 235 & 361 & 358 & \\
\hline Relevé area (m) & $10 \times 10$ & $20 \times 20$ & $50 \times 50$ & $10 \times 10$ & $20 \times 20$ & $10 \times 10$ & $10 \times 10$ & $20 \times 20$ & \\
\hline Slope $\left({ }^{\circ}\right)$ & 20 & 10 & 10 & 10 & & & 30 & & \\
\hline Exposure & $\mathrm{N}$ & $\mathrm{S}$ & $\mathrm{N}$ & $\mathrm{S}$ & & & $S$ & & \\
\hline Density of layer a (\%) & 5 & & & & & & 5 & & \\
\hline Density of layer b (\%) & 70 & 90 & 10 & 60 & 80 & 60 & 60 & 80 & \\
\hline Cover of layer c (\%) & 20 & 5 & 80 & 20 & 10 & 40 & 40 & 20 & \\
\hline Cover of layer d (\%) & 10 & 5 & 10 & 5 & 10 & 5 & 10 & 5 & \\
\hline Number of species in relevé & 10 & 9 & 15 & 14 & 18 & 18 & 22 & 17 & \\
\hline \multicolumn{10}{|l|}{ D. of community } \\
\hline Cytisus scoparius & 4 & 5 & 4 & 5 & 5 & 3 & 4 & 4 & $\mathrm{~V}$ \\
\hline \multicolumn{10}{|l|}{ Accompanying species: } \\
\hline \multicolumn{10}{|c|}{ a) Cl. Calluno-Ulicetalia et Nardo-Callunetea: } \\
\hline Calluna vulgaris & 3 & + & 2 & + & & 2 & + & & IV \\
\hline Carex ericetorum & & & 1 & + & 1 & 1 & + & & IV \\
\hline Hieracium pilosella & 1 & 1 & 2 & + & 2 & 1 & 2 & & $\mathrm{~V}$ \\
\hline Viola canina & & & + & & & & & & I \\
\hline Pohlia nutans & & & & & & + & + & & II \\
\hline Dicranum scoparium & & & & & & & & + & I \\
\hline Cladonia coniocraea & & & & + & + & + & & & II \\
\hline Cladonia deformis & & & & & & + & & & I \\
\hline
\end{tabular}




\begin{tabular}{|c|c|c|c|c|c|c|c|c|c|}
\hline Relevé number in table & 1 & 2 & 3 & 4 & 5 & 6 & 7 & 8 & Constancy \\
\hline \multicolumn{10}{|c|}{ b) Cl. Epilobietea angustifolii: } \\
\hline Calamagrostis epigejos & 1 & 1 & 3 & 2 & 2 & 1 & 2 & 3 & $\mathrm{~V}$ \\
\hline \multicolumn{10}{|c|}{ c) Cl. Koelerio glaucae-Corynephoretea canescentis: } \\
\hline Corynephorus canescens & + & + & 2 & 1 & 1 & 1 & + & + & $\mathrm{V}$ \\
\hline Spergula morisonii & & & + & & 1 & & & & II \\
\hline Helichrysum arenarium & & & & + & & + & & + & II \\
\hline Potentilla arenaria & & & & & & & & + & I \\
\hline Thymus serpyllum & & & & & 1 & & & + & II \\
\hline Rumex acetosella & & & & & & + & + & & II \\
\hline Jasione montana & & & & & + & + & 1 & & II \\
\hline $\begin{array}{l}\text { Lotus corniculatus var. } \\
\text { crassifolius }\end{array}$ & & & & & 1 & & & & $\mathrm{I}$ \\
\hline Scleranthus perennis & & & & & & + & & & I \\
\hline Hypohoeris radicata & & & & & & & + & & I \\
\hline Brachythecium albicans & + & & + & & & & & & II \\
\hline Ceratodon purpureus & + & & + & & & & & & II \\
\hline Polytrichum piliferum & + & + & & + & + & & + & + & IV \\
\hline Cladonia uncialis & & & & & + & & & + & II \\
\hline Cladonia macilenta & & & & & + & + & & & II \\
\hline \multicolumn{10}{|c|}{ d) Cl. Festuco-Brometea et Trifolio-Geranietea: } \\
\hline Galium verum & & & & & & & & 1 & I \\
\hline Dianthus carthusianorum & & & & & 1 & + & 1 & + & III \\
\hline \multicolumn{10}{|l|}{ e) Cl. Vaccinio-Piceetea: } \\
\hline Pinus sylvestris $b$ & & & 1 & + & & & & & II \\
\hline Peucedanum oreoselinum & 1 & & + & + & + & & + & 2 & IV \\
\hline Solidago virgaurea & 2 & + & + & + & 1 & 1 & + & + & $\mathrm{V}$ \\
\hline Deschampsia flexuosa & & & 1 & & & & 1 & & II \\
\hline Cladonia fimbriata s. 1. & & + & & + & & & + & + & III \\
\hline Cladonia gracilis & & & & + & & & + & + & II \\
\hline Calamagrostis arundinacea & & & & & & & 1 & & I \\
\hline \multicolumn{10}{|c|}{ f) Cl. Molinio-Arrhenatheretea: } \\
\hline Rumex acetosa & & & & & & & + & & I \\
\hline Molinia caerulea & & & & & & 1 & & & I \\
\hline Knautia arvensis & & & & & + & & & & I \\
\hline \multicolumn{10}{|l|}{ g) other species: } \\
\hline Betula pendula a & & & & & & & 1 & & I \\
\hline Betula pendula $\mathrm{c}$ & & & + & & & & & & I \\
\hline Quercus petreae c & & & & & & & + & & I \\
\hline Hypericum perforatum & & & & & & + & + & + & II \\
\hline Polytrichum juniperinum & & + & & & + & & & + & II \\
\hline
\end{tabular}


of southern, less frequently western exposure. The association is characterized by the domination of the tussock grass Corynephorus canescens, which is accompanied by Spergula vernalis. It is a pioneer community, very scattered, floristically poor, which initiates the process of secondary overgrowing of sandy dunes (Namura-Ochalska 2004). Between tussocks of gray clubawn grass, bare sand occurs, on which annual species appear in spring, including previously mentioned Spergula vernalis, which vanishes just after seeding. In the more advanced developmental phases, when the substratum is more and more stable, there begin to appear smaller or bigger clusters of lichens, mostly from the genus Cladonia and mosses, mainly Polytrichum piliferum. The frequent presence of heath plants, such as: Hieracium pilosella and Calluna vulgaris, in the composition of the described community is its significant characteristic. As compared to typical dry heaths in the described association, the latter species occurs less abundantly and usually on the outskirts. Also species differential for coniferous forests from the class Vaccinio-Piceetea, constitute a component of Corynephorus grasslands in the studied area (Fig. 4). The occurrence of trees - Scots pine Pinus sylvestris and European white birch Betula pendula is a frequent phenomenon.

The association Calamagrostietum epigeji, classified within clearing communities from the class Epilobietea angustifolii, border on grasslands of gray clubawn grass or heathlands. However, it is a relatively rare community in the training ground. It forms small patches in flat areas, less frequently on slopes of very small inclination. Most frequently its phytocoenoses border on grasslands of gray clubawn grass or heathlands. In this association from the studied area, two forms were distinguished: typical and with Vincetoxicum hirundinaria (Tab. 5).

\subsubsection{Pine forests and groves of birch and aspen trees}

At present, in the studied part of the artillery range near Toruń, heaths and psammophilous grasslands dominate, however, in many places the landscape is diversified by different size fragments of developing forests, which are at several different developmental phases. Among them, communities dominated by pine predominate. Also a large area is covered by groves of birch and aspen trees, as well as birch and pine forests. Their age is diversified and ranges from 5 to 65 years. The majority of tree stands are at the age of 21-30 years. Moreover, a small area is covered by forests with some contribution of oak and hazel thickets. In phytosociological respect, pine forests from the class Vaccinio-Piceetea predominate in the studied area. Small areas are covered by acidophilous oak forest from the class Quercetea robori-petraea and thickets of mixed lime-oak-hornbeam forests from the class QuercoFagetea.
Melkowska (2009) stated that pine forests of the studied area belong to the subcontinental association PeucedanoPinetum, which differentiates here into three variants: the variant with Geranium sanguineum, the typical and the poor variant. A different number of species occur in them. The variant with Geranium sanguineum is the richest in species. On average, 29 species of vascular plants, bryophytes and lichens occur in a relevé of $400 \mathrm{~m}^{2}$. In the typical and the poor variant, the average number of species amounts to 22 and 16 respectively.

Out of three variants, the variant with Geranium sanguineum is the most diverse one in respect of species composition. Within it, one can distinguish two subvariants: with Molinia caerulea and the typical one.

Melkowska (2009) distinguishes the following syntaxa among deciduous forests: the community with Corylus avellana in the habitat of mixed lime-oak-hornbeam forest Tilio-Carpinetum from the class Querco-Fagetea, the association Calamagrostio arundinaceae-Quercetum petraeae from the class Quercetea robori-petraeae, as well as the community with Populus tremula and the community with Betula pendula in the habitat of pine forest PeucedanoPinetum.

\section{Syndynamic tendencies of vegetation from the training ground and discussion of the results}

In the analysed set of relevés and among the distinguished communities of psammophilous grasslands and dry heaths occurring on the training ground near Torun, one can observe phytocoenotic structures proving the extinction of certain types of communities and their transformation into other communities, which are the subsequent stage of secondary succession. This succession is manifested by the presence of early phases of encroachment of species from psammophilous grasslands or kinnikinnick bearberry Arctostaphylos uva-ursi on dune quicksand. Together with „maturation" of psammophilous grasslands and bearberry heaths, on their patches there encroach species initiating the further development of a phytocoenosis towards heaths of nodding thread-moss Pohlio-Callunetum, the association of chee reedgrass Calamagrostietum epigeji, or the community with common groom Cytisus scoparius. The last stage of secondary succession consists in the encroachment of tree species and transformation of non-forest communities into birch forests, as well as pine and mixed forests.

In the studied area, the association Spergulo vernalisCorynephoretum occurs both in the early stages of succession, as well as in the subsequent developmental phases. In the pioneer Corynephorus grasslands, the layer of mosses and lichens is poorly developed, which can be observed in relevés $4,13,14,15$ in the Table 4 . The average number 
of species per relevé amounts to 11 . As the contribution of gray clubawn grass increases and the substratum stabilizes, the number of species and, at the same time, the diversity of a community increases. It is the subsequent phase of succession, in which mosses begin to occur in more humid places, whereas dry and insolated sites are taken over by lichens. Then, heather and pine seedlings encroach. In the still more advanced stages of the development, young specimens of trees and shrubs are highly significant, the layer of mosses and lichens is already very well developed and the number of species per relevé reaches 22 (relevés 2, 5, 6, Tab. 4). Ultimately, the community assumes the features of the association Pohlio-Callunetum.

Based on the analysis of floristic composition and comparisons of developmental phases of the association Spergulo vernalis-Corynephoretum, one can state that it is an impermanent community, susceptible to processes of secondary succession. It develops in insolated areas and therefore it is rich in photophilous species. Artificial planting of pine, as well as spontaneous encroachment of shrubby and arborescent species from neighbouring pine forests, trigger off the processes of secondary succession leading to transformations of Corynephorus grasslands into the communities of dwarf shrubs and thickets, followed by forest communities. In other regions of Poland, succession transformations of the association Spergulo vernalis-Corynephoretum may lead towards communities from the class Festuco-Brometea and Molinio-Arrhenatheretea, whereas in the case of Corynephorus grasslands from the artillery range near Torun, the succession leads towards reconstruction of pine forests.

In the studied area, heath patches of Arctostaphylo-Callunetum, similarly like those of the association Spergulo vernalis-Corynephoretum, are not durable phytocoenoses. They are subject to perceivable transformations of the structure, despite their occurrence in extreme habitat and thermal conditions, with the disturbance resulting from military operations, which restrains or delays the succession processes.

Patches of the association Arctostaphylo-Callunetum, where kinnikinnick bearberry Arctostaphylos uva-ursi dominates, are pioneer succession stages. Those patches cover highly insolated areas on the tops of dunes, where thermal and soil conditions are favourable for photophilous species. Patches of this association, recorded in the studied area, with slightly different structure, are characterized by significantly lower contribution of Arctostaphylos uva-ursi and the domination of Calluna vulgaris, as well as high contribution of coniferous-forest species, such as Pinus sylvestris or Deschampsia flexuosa. Low contribution of bearberry proves its retreat from areas dominated by heather. This process causes transformations of the association Arctostaphylo-Callunetum into heaths of PohlioCallunetum.
When describing the biology and dynamics of bearberry and bearberry heaths, Ciosek (2000) paid attention to the results of the research by Hobbs (1984), who studied the influence of extracts of some plant species occurring in heaths on other species, as well as quantitative relationships between those species. The results revealed harmful influence of bearberry on the germination and the growth of Calluna vulgaris. Based on his own research carried out on heathlands from eastern Poland, Ciosek (2000) stated that in most cases the range lines of Arctostaphylos uvaursi and Calluna vulgaris do not overlap. From this it appears that in the tussocks of bearberry heaths Arctostaphylo-Callunetum, bearberry and heather generally exclude each other.

A similar relationship becomes apparent from the research carried out on the artillery range near Torun. When the cover of Calluna vulgaris exceeds $25 \%$, the occurrence of Arctostaphylos uva-ursi is low. Whereas, in the patches where percentage cover of the heather is below $25 \%$, the density of bearberry is evidently higher and the species dominates in these areas. Additionally, in places dominated by bearberry, heather persists only on the edges of bearberry patches and it does not encroach into their interior parts.

The association of Pohlio-Callunetum is also subject to succession processes, which is manifested by high diversity of its types and the number of species. Numerous variants, subvariants and forms of this association prove that other communities occurring in its neighbourhood have significant influence on its structure. And thus, it takes on features of both pioneer communities, i.e. Corynephorus grasslands and bearberry heaths, as well as features of the community with Cytisus scoparius, the association Calamagrostietum epigeji and pine forests Peucedano-Pinetum occurring within the training ground. The form of the association with Pleurozium schreberi, both in the variant and in the typical subvariant, proves the encroachment of coniferous-forest species on the heathlands, including tree species, particularly Pinus sylvestris and Betula pendula. Relevés from the form with Pleurozium schreberi were made on the heathlands developing near pine forests Peucedano-Pinetum. The sites with this kind of location are characterized by habitat conditions different from those prevailing in heath patches situated at a further distance from the forest, and where the remaining relevés of the association were made. During the field studies, one could observe that the soil moisture, its acidity and the shading of vegetation in the Pohlia nutans heathlands increased along the gradient from the outskirts of pine forest towards its interior, which was expressed through different species composition, in the form of numerous forest species of mosses and lichens, e.g. Dicranum undulatum, Pleurozium schreberi, Cladonia gracilis, C. rangiferina, C. arbuscula subsp. arbuscula. At the same time, the quantity of Cal- 
luna vulgaris often decreased giving way to species from the class Vaccinio-Piceetea.

Apart from psammophilous grasslands and heaths, also two other communities occur in the studied area of the artillery range, covering much smaller areas, i.e. the association Calamagrostietum epigeji and the community with common groom Cytisus scoparius. The former syntaxon occurs near the grasslands Spergulo vernalis-Corynephoretum and heaths Pohlio-Callunetum. According to Matuszkiewicz (2007), Calamagrostis epigejos is not a very demanding species concerning the habitat conditions. It grows on loose and acid soils, and its strongly expanding stolons choke up other plant species. Probably, when this association encroach on favourable dune areas, covered with grasslands, it contributes to their overgrowing and disappearance. In the studied area of the training ground, only few patches of this association were recorded and due to a small number of documentary materials, comprising only 8 relevés, it is difficult to draw any tangible conclusions concerning the changes and transformations occurring within thereof.

The community with Cytisus scoparius, similarly like the association Calamagrostietum epigeji, covers only small areas in the studied sections of the forest district of Otłoczyn. However, within the whole area of the artillery range, there can be observed fast spreading of Cytisus scoparius, which is related to good habitat conditions of this species. No species from the class Rhamno-Prunetea have been observed in the patches of this community occurring here (Fig. 4).

The presence of species characteristic of Corynephorus grasslands in the phytocoenoses dominated by common broom and in the patches of chee reedgrass, allows to assume that the community with Cytisus scoparius, as well as the association Calamagrostietum epigeji constitute the transitional form of the association Spergulo vernalis-Corynephoretum on the artillery range near the city of Torun.

\section{Conclusions}

1. Plant communities occurring in the studied part of the artillery range constitutes a succession sequence, starting from pioneer Corynephorus grasslands Spergulo vernalisCorynephoretum and bearberry heaths Arctostaphylo-Callunetum, through a transitional stage of heaths with nodding thread-moss Pohlio-Callunetum, or communities with common groom Cytisus scoparius, or the association with chee reedgrass Calamagrostietum epigeji, ending with the final stage of pine forests Peucedano-Pinetum.

2. Communities occurring in the direct, close contact of grasslands and heaths have a strong influence on the direction of succession transformations in the training ground near Toruń. Depending on neighbouring plant communities, species composition and directions of succession transformations change.

3. Psammophilous grasslands and heaths occurring within the artillery range near Torun are anthropogenic and are strictly dependent on the intensity of human activities.

4. Encroachment of shrub and arborescent species reflects less common and less intense exploitation of the training ground in recent years for military trainings. Because operations comprising artillery firing, hand grenade throwing and using heavy technical equipment, especially military vehicles, have a significant influence on the preservation of non-forest communities in the training ground. Periodic fires, brought on by explosions of grenades, shells and missiles, contribute to the destruction of self-seeding shrubs and trees, and at the same time contribute to exposing the soil cover, which is favourable to the development of heliophilous dune vegetation, psammophilous grasslands and patches of Arctostaphylos uva-ursi initiating the development of pioneer bearberry heaths.

5. Cessation of military activities in certain parts of the training ground, as well as the fact that the anthropogenic factor is not really heavily destructive, contributes to disappearance of grassland and heath communities on the training ground through encroachment of forest species and formation of birch, aspen and pine thickets, and then pine forest, which constitutes a subsequent stage of secondary succession.

6 . The conducted research contributed to better appreciation of nature values of this area and induced some actions aiming at its protection. The research validated the efforts to create the Special Area of Conservation of Natura 2000 Habitats within the training ground, called „Dunes of the Toruń Valley".

\section{References}

Braun-Blanquet J., 1951, Pflanzensoziologie-Grundzüge der Vegetationskunde, Wien.

Ciosek M.T., 2000, Mącznica lekarska Arctostaphylos uva-ursi i wrzosowiska mącznicowe ArctostaphyloCallunetum w Polsce środkowowschodniej na tle ich zróżnicowania w Polsce [Bearberry, Arctostaphylos uva-ursi (L.) Spring., and bearberry moors, Arctostaphylo-Callunetum R. Tx. et Prsg 1949, in eastern central Poland in relation to their diversity in Poland], Rozprawa Naukowa 65, WAP, Siedlce.

Dzięgielewski K., 1997, Toruński poligon artyleryjski, Praca dyplomowa napisana w Podyplomowym Studium Polityki Regionalnej [The artillery range in Torun, Thesis written in the Graduate School of Regional Policy], UMK, Toruń. 


\begin{tabular}{|c|c|c|c|c|c|c|c|c|c|c|c|c|c|c|c|c|c|c|c|c|c|c|c|}
\hline 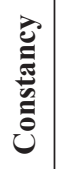 & & & & & & & & & & & & & $>$ & $\Xi$ & $\Xi$ & $\Xi$ & $>$ & $\Xi$ & $\exists$ & $=$ & & - & - \\
\hline$\stackrel{2}{2}$ & $\stackrel{\Xi}{\Xi}$ & 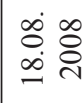 & $\stackrel{\text { g }}{\mathrm{i}}$ & त्ञ్ & ஜ & is & & & \& & 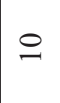 & 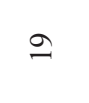 & & r & & + & + & + & & + & & & & \\
\hline$\simeq$ & 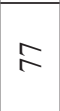 & $\begin{array}{l}0 \\
\text { s. } \\
\end{array}$ & $\underset{\sim}{~}$ & $\begin{array}{l}\text { त्रे } \\
\text { ते }\end{array}$ & $\stackrel{\circ}{\circ}$ & is & in & - & $\infty$ & $\stackrel{i}{\text { i }}$ & $=$ & & r & & - & & & & - & & & & \\
\hline \pm & $\infty$ & $\dot{0} \dot{0}$ & $\ddot{\sim}$ & $\begin{array}{l}\text { त्त्र } \\
\stackrel{\sim}{\tilde{N}}\end{array}$ & 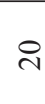 & 3 & & & \& & ¿ి & 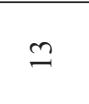 & & 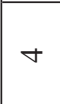 & & & + & - & & + & & & & \\
\hline$\cong$ & 8 & $\begin{array}{l}\dot{0} \infty \\
\dot{b}\end{array}$ & $\approx$ & $\begin{array}{l}\text { ते } \\
\text { त् }\end{array}$ & q & س & & & ฉ & ì & $\Xi$ & & $m$ & & + & & & + & + & & & & \\
\hline$\simeq$ & $\overrightarrow{0}$ & $\begin{array}{l}\dot{0} \\
\dot{0} \\
\dot{\omega}\end{array}$ & $\ddot{\imath}$ & $\stackrel{\circ}{\stackrel{x}{\circ}}$ & & & - & in & $\stackrel{R}{R}$ & ¿ & $\stackrel{\infty}{-}$ & & $m$ & & & + & - & & + & & & & \\
\hline$=$ & $\stackrel{+}{n}$ & $\begin{array}{l}0 \\
0 \\
0\end{array}$ & $\underset{\sim}{\tilde{\lambda}}$ & 音 & 우 & z & in & & $尺$ & $\stackrel{\text { 入 }}{ }$ & $\curvearrowright$ & & $m$ & & + & & + & + & - & + & & & \\
\hline$\Theta$ & $\stackrel{\infty}{+}$ & ic & $\stackrel{+}{\stackrel{\Delta}{\Delta}}$ & 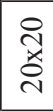 & i & 岀 & & & \& & \& & 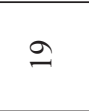 & & $\nabla$ & 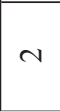 & + & + & - & + & & + & & & \\
\hline$a$ & $\mathscr{f}$ & $\overrightarrow{0} \dot{0}$ & $\underset{\sim}{\infty}$ & 章 & & & - & - & $\triangleright$ & 으 & $\stackrel{\infty}{-}$ & & ナ & & & + & + & + & & & & & \\
\hline$\infty$ & $m$ & $\begin{array}{l}\dot{\delta} \\
\stackrel{\infty}{0} \\
\text { ¿ }\end{array}$ & ڤે & 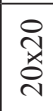 & f & 3 & & $\circ$ & $\infty$ & 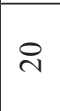 & $=$ & & in & N & - & & - & + & + & & & & - \\
\hline$r$ & i & $\begin{array}{l}\dot{\leftrightarrow} \\
\dot{\lambda} \\
\stackrel{े}{े}\end{array}$ & के & ते & f & 3 & & & 8 & ¿े & 으 & & r & $N$ & & & - & & + & & & & + \\
\hline 0 & $\stackrel{\infty}{\sim}$ & $\begin{array}{l}\dot{\Xi} \\
\text { ¿े } \\
\text { ते }\end{array}$ & ळે & 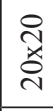 & 으 & in & & 으 & in & i & $\stackrel{\sim}{\sim}$ & & $\nabla$ & & + & & & & & & & - & + \\
\hline in & $\ddot{\imath}$ & 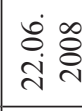 & $\frac{\bar{m}}{}$ & 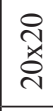 & ¿ & in & & - & 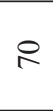 & \& & ส & & $m$ & - & + & + & + & - & + & + & $\ddot{\Xi}$ & - & \\
\hline$\nabla$ & 9 & $\begin{array}{l}\dot{\infty} \\
\infty \\
\infty \\
\infty\end{array}$ & in & 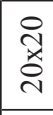 & i & in & & & q & ஓ্ল & $\simeq$ & 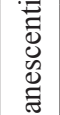 & $n$ & - & + & & + & $\sim$ & & & $\begin{array}{l}\text { : } \\
\overline{2} \\
\overline{0} \\
\text { है }\end{array}$ & & \\
\hline$m$ & $\stackrel{m}{2}$ & $\begin{array}{l}\dot{0} \infty \\
\infty \\
\infty \\
\infty\end{array}$ & 冓 & 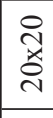 & 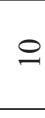 & is & & & 8 & 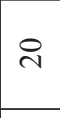 & 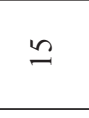 & $\begin{array}{l}0 \\
0 \\
.0 \\
.0 \\
0 \\
0\end{array}$ & $\forall$ & & + & & & - & & + & $\begin{array}{l}\overrightarrow{0} \\
\dot{0} \\
\stackrel{0}{0} \\
0\end{array}$ & & \\
\hline$N$ & $N$ & 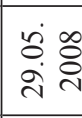 & in & 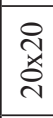 & లి & in & & in & $\triangleright$ & in & 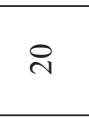 & 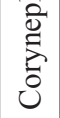 & $m$ & $N$ & & + & + & & & & $\begin{array}{l}\overline{0} \\
\underline{0} \\
\overrightarrow{0} \\
0 \\
0\end{array}$ & & \\
\hline- & - & 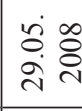 & in & $\underset{\underline{x}}{0}$ & ¿ి & in & & & $R$ & q & $\stackrel{\circ}{\circ}$ & 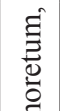 & 防 & $m$ & + & + & - & & & & 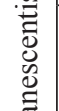 & & \\
\hline 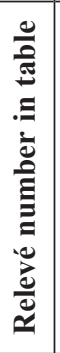 & 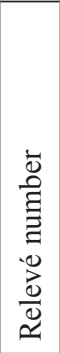 & $\stackrel{\sharp}{\Xi}$ & 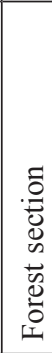 & 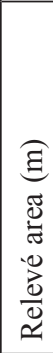 & $\begin{array}{l}0 \\
0 \\
\frac{\partial}{\sigma} \\
\text { n }\end{array}$ & 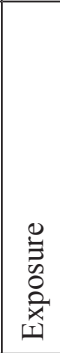 & 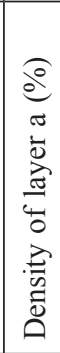 & 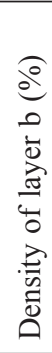 & 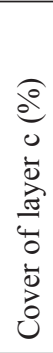 & 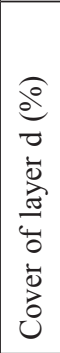 & 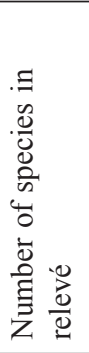 & 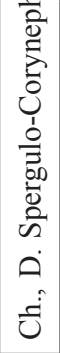 & 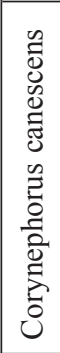 & 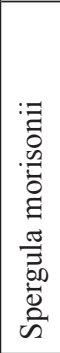 & 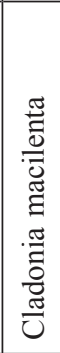 & 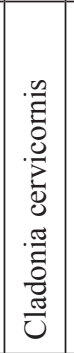 & 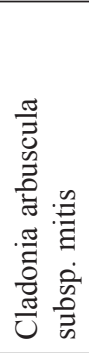 & 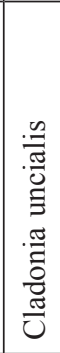 & 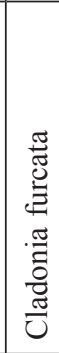 & 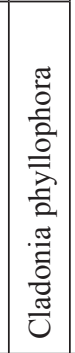 & 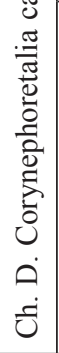 & 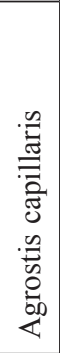 & 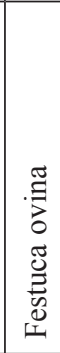 \\
\hline
\end{tabular}




\begin{tabular}{|c|c|c|c|c|c|c|c|c|c|c|c|c|c|c|c|c|c|c|c|c|c|c|c|c|c|}
\hline 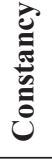 & - & $=$ & - & $=$ & - & - & - & $>$ & $>$ & & & $\Xi$ & $\geq$ & $>$ & - & - & - & - & - & $\Xi$ & $\Rightarrow$ & & $\neg$ & - & - \\
\hline$\underline{\bullet}$ & & & & & & & & - & - & & & + & + & $\sim$ & & & + & & & + & & & + & + & \\
\hline 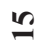 & & & & & & & & & & & & & & $\sim$ & & & & & & & & & & & \\
\hline$\Xi$ & & & & & & & & - & - & & & & + & + & & & & & & + & + & & & & \\
\hline 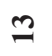 & & - & & & & + & & $N$ & $\sim$ & & & & - & $N$ & & & & & & & + & & & & \\
\hline$\simeq$ & + & + & + & + & & & & - & - & & & - & - & 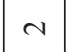 & & & & + & + & + & & & & & \\
\hline$=$ & & & & & & & & - & - & & & - & + & - & & & & & + & + & & & & & \\
\hline$\varrho$ & & & - & & & & & 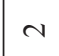 & $\mathrm{N}$ & & & + & + & & & + & & & & & + & & & & \\
\hline$a$ & & & & & & & & + & + & & & - & - & - & + & + & & & & & & & & & \\
\hline$\infty$ & & & & & & & & + & + & & & & & - & & & & & & & & & + & & \\
\hline$r$ & & & & & & + & + & - & - & & & - & - & - & & & & & & & & & + & & \\
\hline 0 & - & + & & & & & & + & + & & & - & - & - & & & & + & & & & & & & + \\
\hline in & & - & & - & + & & & + & + & & & & + & - & & & & & & + & + & & & & \\
\hline$\nabla$ & & & & & & & & + & + & & & + & + & - & & & & & & & & & & & \\
\hline$m$ & & & & + & & & & + & + & & & + & & - & & & & & + & + & + & $\mathbb{E}$ & & & \\
\hline$\sim$ & & - & & & & + & + & $n$ & $m$ & & 总 & & & 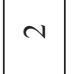 & & & & & & & & 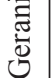 & & & \\
\hline- & & & & + & & & + & - & - & & 离 & & & $N$ & & & & & & + & + & $\stackrel{0}{\Xi}$ & & & \\
\hline 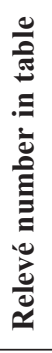 & 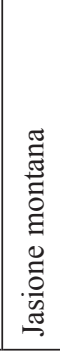 & 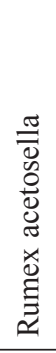 & 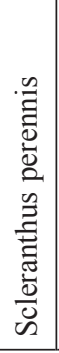 & 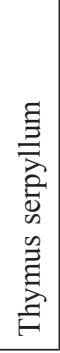 & 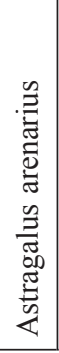 & 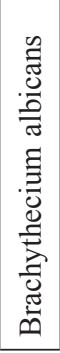 & 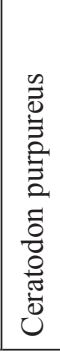 & 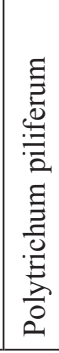 & 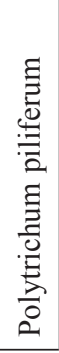 & 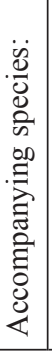 & 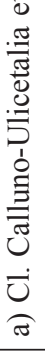 & 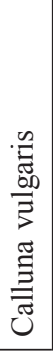 & 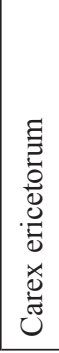 & 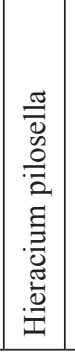 & 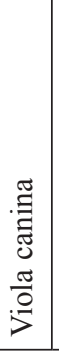 & 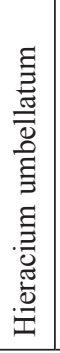 & 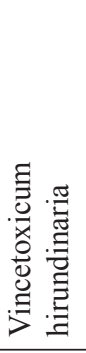 & 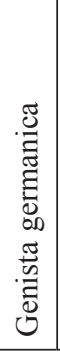 & 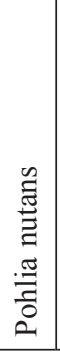 & 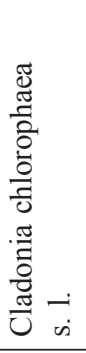 & 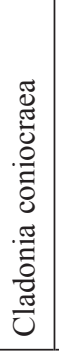 & 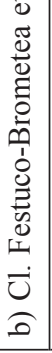 & 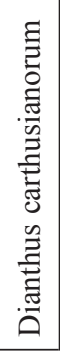 & 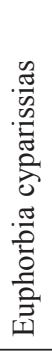 & 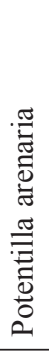 \\
\hline
\end{tabular}




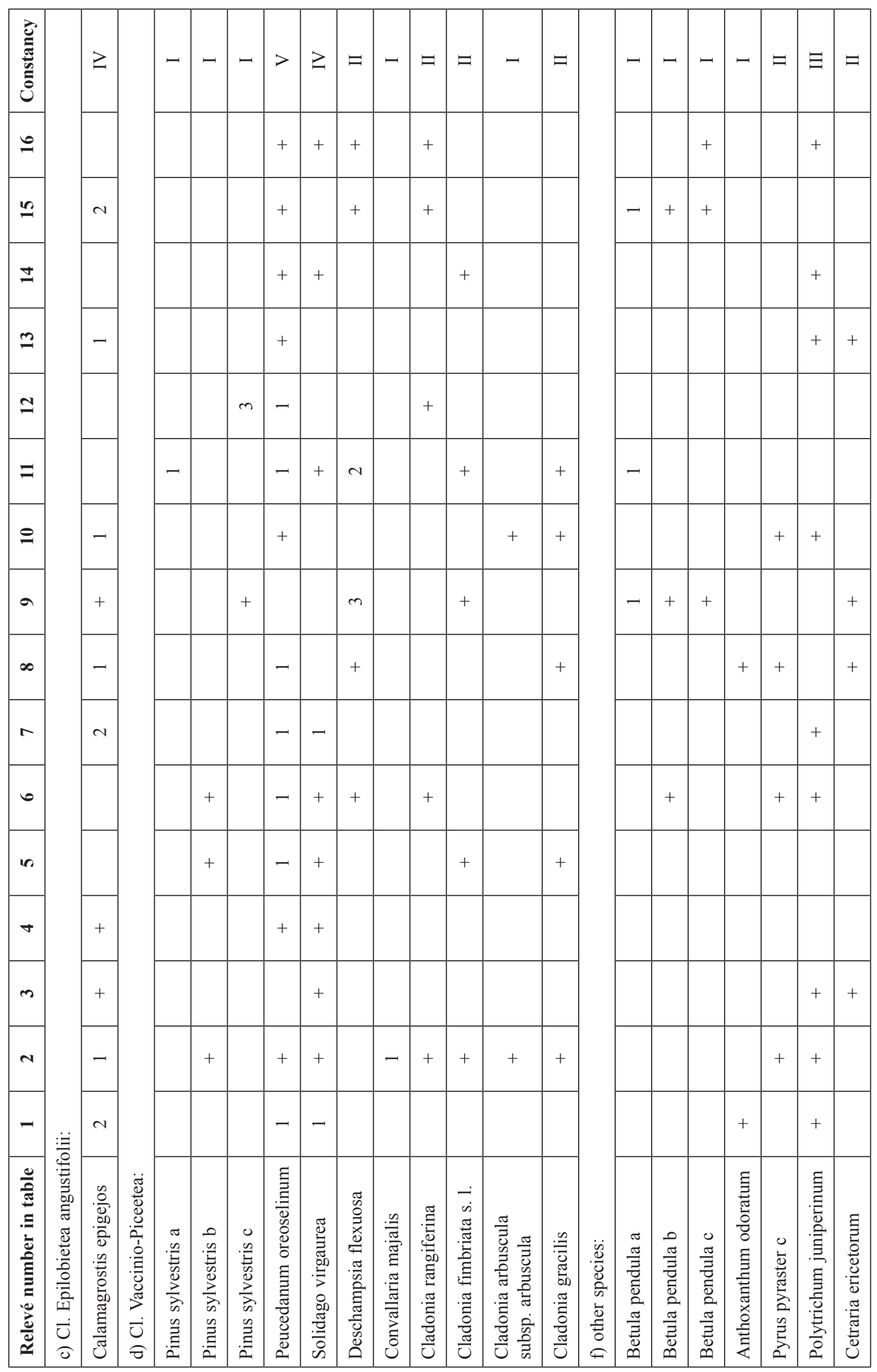


Table 5. The association Calamagrostietum epigeji (relevés 1-5, the typical form; relevés 6-8, the form with Vincentoxicum hirundinaria)

\begin{tabular}{|c|c|c|c|c|c|c|c|c|c|}
\hline Relevé number in table & 1 & 2 & 3 & 4 & 5 & 6 & 7 & 8 & Constancy \\
\hline Relevé number & 16 & 17 & 106 & 100 & 67 & 80 & 110 & 105 & \\
\hline Date & $\begin{array}{c}18.06 \\
2008\end{array}$ & $\begin{array}{c}18.06 \\
2008\end{array}$ & $\begin{array}{c}18.08 \\
2008\end{array}$ & $\begin{array}{c}18.08 \\
2008\end{array}$ & $\begin{array}{c}16.07 \\
2008\end{array}$ & $\begin{array}{c}17.07 . \\
2008\end{array}$ & $\begin{array}{c}18.08 \\
2008\end{array}$ & $\begin{array}{c}18.08 \\
2008\end{array}$ & \\
\hline Forest section & 358 & 358 & 240 & 279 & 235 & 236 & 240 & 241 & \\
\hline Relevé area (m) & $10 \times 10 \mathrm{~m}$ & $10 \times 10$ & $10 \times 10$ & $20 \times 20$ & $10 \times 10$ & $10 \times 10$ & $10 \times 10$ & $10 \times 10$ & \\
\hline Slope $\left({ }^{\circ}\right)$ & 10 & & & & & & & & \\
\hline Exposure & S & & & & & & & & \\
\hline Density of layer a (\%) & & & & & 1 & & & & \\
\hline \multicolumn{9}{|l|}{ Density of layer b (\%) } & \\
\hline Cover of layer c (\%) & 90 & 70 & 90 & 90 & 90 & 90 & 90 & 90 & \\
\hline Cover of layer d (\%) & 5 & 10 & 10 & & 5 & 5 & 10 & 10 & \\
\hline Number of species in relevé & 12 & 15 & 14 & 11 & 13 & 16 & 22 & 23 & \\
\hline \multicolumn{10}{|l|}{ Ch. Calamagrostietum epigeji } \\
\hline Calamagrostis epigejos & 5 & 4 & 5 & 5 & 3 & 4 & 4 & 5 & $\mathrm{~V}$ \\
\hline \multicolumn{10}{|c|}{ Ch. D. Epilobion angustifolii et Epilobietea angustifolii: } \\
\hline Cytisus scoparius & + & & & & & & & + & II \\
\hline Rubus idaeus & & & & 2 & & & & & I \\
\hline Fragaria vesca & & & & & & & & + & I \\
\hline Rumex acetosella & & & & & & + & + & & II \\
\hline \multicolumn{10}{|l|}{ Accompanying species: } \\
\hline \multicolumn{10}{|c|}{ a) Cl. Calluno-Ulicetalia et.Nardo-Callunetea: } \\
\hline Calluna vulgaris & 2 & + & + & + & + & & 1 & + & $\mathrm{V}$ \\
\hline Carex ericetorum & & 1 & + & & 1 & + & & + & IV \\
\hline Hieracium pilosella & + & 1 & 1 & & 1 & 2 & 1 & 1 & $\mathrm{~V}$ \\
\hline Viola canina & & & & & & & + & + & II \\
\hline Vincetoxicum hirundinaria & & & & & & + & 1 & 1 & II \\
\hline Hieracium umbellatum & & & & & & + & & & I \\
\hline Genista tinctoria & 1 & & & & & & & & I \\
\hline Dicranum scoparium & & & & & & & + & & I \\
\hline Cladonia coniocraea & & + & & & & & & + & II \\
\hline Cladonia chlorophaea s. 1. & + & + & + & & & + & + & & IV \\
\hline Cladonia deformis & & & & & + & & & & I \\
\hline \multicolumn{10}{|c|}{ b) Cl. Koelerio glaucae-Corynephoretea canescentis: } \\
\hline Corynephorus canescens & & 1 & + & & 1 & 1 & 2 & + & IV \\
\hline Thymus serpyllum & + & + & + & & & & 1 & 1 & IV \\
\hline
\end{tabular}




\begin{tabular}{|l|l|l|l|l|l|l|l|l|l|}
\hline \multicolumn{1}{|c|}{ Relevé number in table } & $\mathbf{1}$ & $\mathbf{2}$ & $\mathbf{3}$ & $\mathbf{4}$ & $\mathbf{5}$ & $\mathbf{6}$ & $\mathbf{7}$ & $\mathbf{8}$ & Constancy \\
\hline Helichrysum arenarium & & & & & & + & + & + & II \\
\hline Potentilla arenaria & & & & & & & & 1 & I \\
\hline Festuca ovina & & + & & & & & & & I \\
\hline Jasione montana & & + & & & & & & & I \\
\hline Lathyrus sylvestris & & & & + & & & & & I \\
\hline Polytrichum piliferum & & & & & & & + & + & II \\
\hline Cladonia furcata & & & & & + & & & & I \\
\hline
\end{tabular}

c) Cl. Festuco-Brometea et Trifolio-Geranietea:

\begin{tabular}{|l|l|l|l|l|l|l|l|l|r|}
\hline Dianthus carthusianorum & + & & + & & & 1 & + & + & IV \\
\hline Euphorbia cyparissias & & & & & & & + & 2 & II \\
\hline Achillea pannonica & & & & & & & + & 1 & II \\
\hline Galium verum & & & & & + & & & & I \\
\hline Anthericum ramosum & & & & & + & & & & I \\
\hline
\end{tabular}

d) Cl. Molinio-Arrhenatheretea:

\begin{tabular}{|l|l|l|l|l|l|l|l|l|l|}
\hline Molinia caerulea & & 1 & & & + & + & 1 & & III \\
\hline Rumex acetosa & & & & 2 & & & & & I \\
\hline Achillea millefolium & & & & & & & & + & I \\
\hline
\end{tabular}

e) Cl. Vaccinio-Piceetea:

\begin{tabular}{|c|c|c|c|c|c|c|c|c|c|}
\hline Solidago virgaurea & + & 1 & 1 & + & + & + & 1 & + & $\mathrm{V}$ \\
\hline Peucedanum oreoselinum & & + & + & & & & + & + & III \\
\hline Deschampsia flexuosa & + & & & 2 & & & + & & II \\
\hline Pinus sylvestris c & & & & & + & & + & & II \\
\hline $\begin{array}{l}\text { Cladonia arbuscula subsp. } \\
\text { arbuscula }\end{array}$ & & & & & + & + & + & & II \\
\hline Cladonia fimbriata s. 1. & & & + & & & + & & + & II \\
\hline \multicolumn{10}{|l|}{ f) other species: } \\
\hline Betula pendula a & & & & & 1 & & & & I \\
\hline Betula pendula $\mathrm{c}$ & & & & 1 & & & & & I \\
\hline Hypericum perforatum & & + & + & & & 1 & + & + & IV \\
\hline Linaria vulgaris & & & & 1 & & & + & 1 & II \\
\hline Anthoxanthum odoratum & + & & & & & & & & I \\
\hline Pyrus communis $\mathrm{c}$ & & + & & & & & & & I \\
\hline Carduus crispus & & & & + & & & & & I \\
\hline Campanula rotundifolia & & & & & & & & + & I \\
\hline
\end{tabular}


Galon R., 1958, Z problematyki wydm śródlądowych w Polsce, tom 1 [Sur les dunes continentales en Polotne, vol. 1], [in:] R. Galon (ed.) Wydmy śródlądowe Polski [Inland Sand Dunes of Poland], PWN, Warszawa: 13-31.

Gawłowska J., 1964, Mącznica lekarska - Arctostaphylos uva-ursi L. w Polsce, jej zasoby i ochrona [Bearberry, Arctostaphylos uva-ursi L., in Poland, its resources and protection], Ochrona Przyrody 30: 23-50.

Hobbs R. J., 1984, Possible chemical interactions among heathland plants, Oikos 43(1): 23-29.

Kejna M., 2009, Dane klimatyczne ze Stacji Bazowej Zintegrowanego Monitoringu Środowiska w Koniczynce za lata 1994-2008, materiały niepublikowane [Climatic data from the Base Station of Integrated Environmental Monitoring in the village of Koniczynka for the years 1994-2008, unpublished materials], UMK, Torun.

Kobendza J. \& Kobendza R., 1958, Rozwiewane wydmy Puszczy Kampinoskiej, tom 1 [Les dunes éparpillées de la Forét de Kampinos, vol. 1], [in:] R. Galon (ed.) Wydmy śródlądowe Polski [Inland sand dunes of Poland], PWN, Warszawa: 95-170.

Kondracki J., 2000, Geografia regionalna Polski [Regional geography of Poland], PWN, Warszawa.

Kujawa-Pawlaczyk J., 2004, Suche wrzosowiska (CallunoGenistion, Pohlio-Callunion, Calluno-Arctostaphylion) [Dry heathlands (Calluno-Genistion, Pohlio-Callunion, Calluno-Arctostaphylion)], [in:] J. Herbich (ed.) Murawy, łąki, ziołorośla, wrzosowiska, zarośla, Poradniki ochrony siedlisk i gatunków Natura 2000 - podręcznik metodyczny, tom 3 [Grasslands, meadows, tall herb vegetation, heaths, thickets, Guides to conservation of habitats and species Natura 2000 - The methodical guidebook, Volume 3], Ministerstwo Środowiska, Warszawa: 32-48.

Matuszkiewicz J. M., 1993, Krajobrazy roślinne i regiony geobotaniczne Polski, Prace geograficzne Nr 158 [Vegetation landscape and geobotanical regions of Poland, Geographical studies No 158] PAN, Inst. Geografii i Przestrzennego Zagospodarowania, Zakład Narodowy im. Ossolińskich, Wrocław - Warszawa - Kraków.
Matuszkiewicz W., 2007, Przewodnik do oznaczania zbiorowisk roślinnych Polski [A guide for identification of plant communities in Poland], PWN, Warszawa.

Melkowska M., 2009, Kształtowanie się zbiorowisk leśnych na poligonie artyleryjskim pod Toruniem [Development of forest plant communities on the artillery range near the city of Toruń], Praca magisterska, Uniwersytet Mikołaja Kopernika, Wydział BiNoZ, Zakład Ekologii Roślin i Ochrony Przyrody [Master's Thesis, Nicolaus Copernicus University, Faculty of Biology and Earth Sciences, Department of Plant Ecology and Nature Protection], UMK, Torun.

Namura-Ochalska A., 2003, Wydmy śródlądowe z murawami napiaskowymi [Inland dunes with arenaceous grasslands], [in:] J. Herbich (ed.) Siedliska morskie i przybrzeżne, nadmorskie i śródlądowe solniska i wydmy, Poradniki ochrony siedlisk i gatunków Natura 2000 - podręcznik metodyczny, tom 1 [Marine and coastal habitats; coastal and inland salt marshes and dunes, Guides to conservation of habitats and species Natura 2000 - The methodical guidebook, Volume 1], Ministerstwo Środowiska, Warszawa: 191-195.

Operat glebowo-siedliskowy. Nadleśnictwo Gniewkowo, Obręb Otłoczyn, 2000, Regionalna Dyrekcja Lasów Państwowych w Toruniu [Survey of soils and habitats, the Forest Division of Gniewkowo, the District of Otłoczyn, 2000, Regional Administration of State Forests in Toruń], RDLP, Toruń.

Wasilewski S., 2004, Zarys historii toruńskiego poligonu artyleryjskiego [An outline history of the artillery range in the city of Toruń], Centrum Szkolenia Artylerii i Uzbrojenia w Toruniu [The Centre for Artillery and Weaponry Training in Toruń], CSzAiU, Toruń.

Werdyn L., 1964, Materiały do rozmieszczenia Arctostaphylos uva-ursi L. na Niżu Polskim [Notes on distribution of Arctostaphylos uva-ursi L. in the Polish Lowlands], Bad. Fizjogr. nad Polską Zach. 14: 127-143.

Wilkoń-Michalska J., 1964, Szata roślinna Kujaw, Przewodnik florystyczny [Vegetation cover of the Kujawy region, Floristic guidebook], TNT, Toruń. 
